\title{
Endosomal proteins NPC1 and NPC2 at African swine fever virus entry/fusion
}

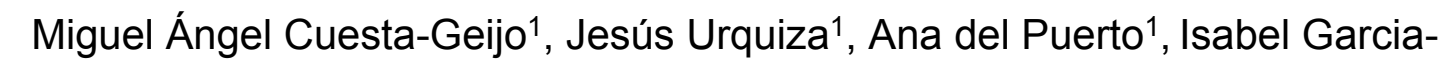
Dorival $^{1}$, Fátima Lasala², Lucía Barrado-Gil ${ }^{1}$, Inmaculada Galindo1', Rafael Delgado $^{2}$ and Covadonga Alonso ${ }^{1 *}$

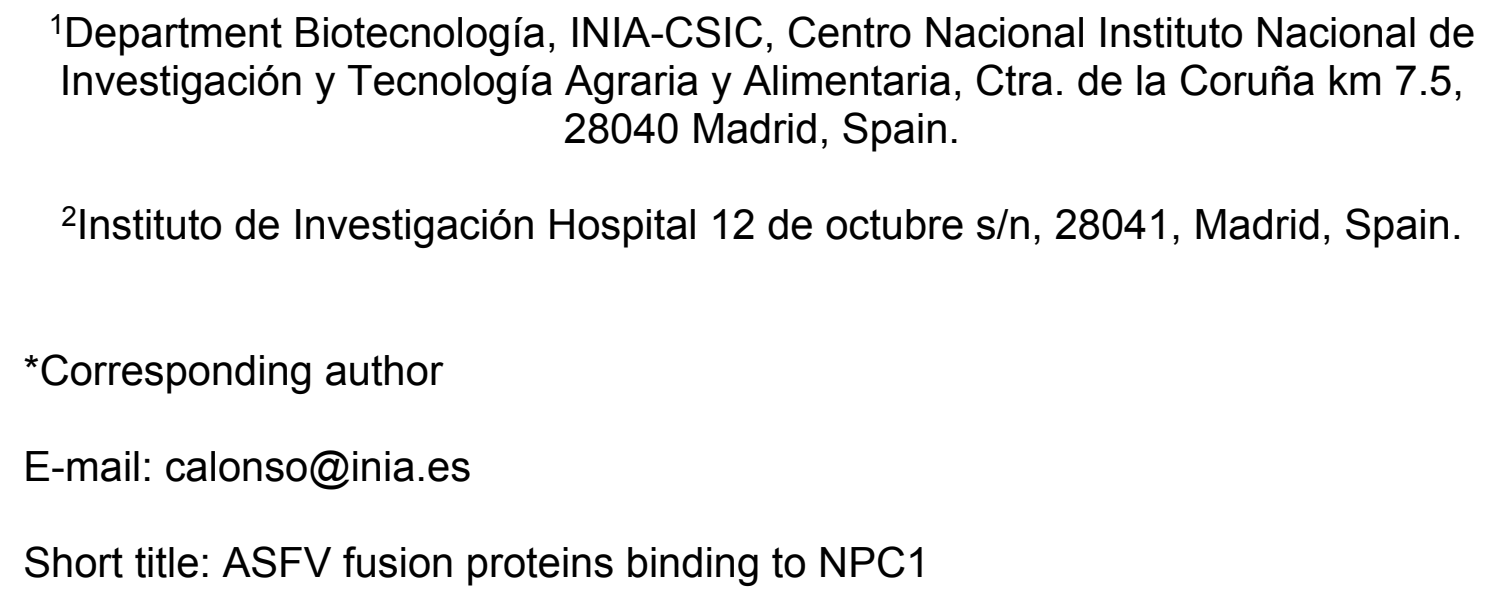
Investigación y Tecnología Agraria y Alimentaria, Ctra. de la Coruña km 7.5, 28040 Madrid, Spain.

2Instituto de Investigación Hospital 12 de octubre s/n, 28041, Madrid, Spain.

${ }^{*}$ Corresponding author

E-mail: calonso@inia.es

Short title: ASFV fusion proteins binding to NPC1

\section{ABSTRACT}

African swine fever virus (ASFV) infectious cycle starts with the viral adsorption and entry into the host cell. The virus is internalized via clathrin/dynamin mediated endocytosis and macropinocytosis. As several other viruses, ASF virion is then internalized and incorporated into the endocytic pathway. Endosomal maturation entails luminal acidification and the lowering of $\mathrm{pH}$ acting on the multi-layered virion structure dissolves the outer capsid. Upon decapsidation, the inner viral membrane is exposed to interact with the limiting membrane of the late endosome for fusion. Egress from endosome is related to cholesterol efflux, but it remains an intriguing process albeit essential for infection, specifically for the viral nucleic acid exit to the cytoplasm for replication. ASFV proteins E248R and 
33 E199L, with structural homology to the VACV proteins of the fusion complex, seem to have similar functions in ASFV. A direct interaction between these ASFV proteins with the cholesterol transporter protein NPC1 (Niemann-Pick C type 1) was observed, which was also shared by the E248R homologous protein L1R of VACV. Binding occurs between the transmembrane domain of E248R with the loop C of NPC1 at the same domain than EBOV binding site. These interactions suggest that these ASFV proteins are crucial for membrane fusion. CRISPR NPC1 KO Vero cells lacking NPC1 protein that were resistant to EBOV, reduced ASFV infection levels significantly. Reductions on ASFV infectivity and replication in NPC1 KO cells were accompanied by lesser viral factories of smaller size and lacking the typical cohesive morphology between endosomes and viral proteins. We observed a compensatory effect in NPC1 KO cells, elevating NPC2 levels while silencing NPC2 in Vero cells with shRNA, also reduced ASFV infection. Our findings pave the way to understand the role of these proteins at the membrane viral fusion step for several viruses.

\section{Author Summary}

African swine fever virus (ASFV) causes a deadly disease of pigs and wild boars that was endemic in Africa but have extended over the last years to Europe, Asia and Oceania with high socioeconomic impact. ASFV enters the cell by endocytosis and has adapted to the endosomal conditions to acquire infectivity. Viral infectivity is dependent on cholesterol traffic at the endosomes, especially at the fusion step. Fusion of the internal viral membrane with the endosomal membrane is required for the exit of the viral DNA to the cytoplasm to start replication. ASF virion internal membrane proteins E248R and E199L were found to bind the Niemann Pick C1 (NPC1) receptor at the endosome. These proteins 
are highly conserved among ASFV isolates and resemble proteins of the VACV entry/fusion complex. The function of NPC1 is to regulate the efflux of dietary cholesterol efflux from the endosome to the endoplasmic reticulum, which appears to be necessary for viral fusion. NPC1 knockout cells by CRISPR reduced infection affecting infectivity and early replication. Also, removing the associated endosomal protein NPC2, further declined infectivity. These results show the relevance of NPC1 receptor in the viral infection actually shared by unrelated important viral families.

\section{INTRODUCTION}

African Swine Fever Virus (ASFV) is the only known member of the Asfarviridae family, and the only known DNA arbovirus. It is a large, enveloped virus with an average diameter of $200 \mathrm{~nm}$ and a multi-layered structure and icosahedral morphology that has been recently unveiled in detail (1-3).

ASFV is the causative agent of African Swine Fever (ASF), a high mortality haemorrhagic disease affecting swine which is endemic in sub-Saharian Africa. However, ASF epidemics that started in the Caucasus and Russian Federation in 2007 (4), have expanded rapidly over different countries in Europe, Asia and Oceania; causing a devastating burden in the global pig industry (5). Currently, ASF cases have been reported in Germany since September 2020 (6); furthermore, India has also recently declared an $\operatorname{ASF}$ outbreak $(7,8)$.

ASFV infectious cycle starts with the viral adsorption and entry into the host cell. After attachment to an unknown receptor, the virus is mainly internalized via clathrin/dynamin mediated endocytosis and micropinocytosis $(9,10)$. Then, the virion is internalized and incorporated into the endocytic pathway. 
87 Under the molecular cues of endosomes, the multi-layered virion undergoes

88 uncoating starting from decapsidation (11). This step will be followed by a less

89 known fusion process at late endosomes (LE), delivering the naked viral core out

90 to the cytoplasm.

91 According to (11), endosomal maturation entails dropping of luminal $\mathrm{pH}$ and this

92 acidic environment disrupts the structure of the virion and produces

93 decapsidation at $30-45$ minutes post infection (mpi). The consequence is the

94 exposure of the inner viral membrane allowing its fusion with the limiting

95 membrane of the LE. This fusion is strongly dependent on cholesterol efflux at

96 the LE. In fact, blocking cholesterol transport at this level causes retention of

97 virions inside endosomes, and inhibits infection progression (12).

98 Cholesterol transport from LE requires the acidic $\mathrm{pH}$ (13) and it is mainly

99 regulated by the coordinated action of LE proteins Niemann Pick type C 1

100 (NPC1), an endolysosomal membrane protein; and NPC2, a single domain

101 protein located in the lumen of the vesicle (14-16).

102 Similar to other viruses, such as Ebola virus (EBOV) $(17,18)$, the ASFV

103 replication cycle is strongly impacted by compounds such as U18666A or

104 imipramine $(19,20)$. These compounds act either by binding to NPC1 sterol sensing domain (SSD) or by blocking EBOV Glycoprotein (GP) GP-NPC1

106 interaction, respectively. This indicates that NPC1 and intact cholesterol transport

107 are pivotal for a successful infection (21-32).

108 Since the underlying mechanisms of viral fusion and egress to the cytoplasm still 109 remain obscure, we hypothesized that ASFV could penetrate to the cytoplasm

110 from LE in a NPC1-dependent manner via proteins belonging to its fusion

111 machinery. 
112 ASFV proteins potentially involved in fusion would be probably internal

113 membrane proteins E248R and E199L. These are structural proteins, late

114 synthesized, which have been involved in ASF fusion and viral core penetration

115 (33-35), lacking a demonstrated interaction between them (34). As both proteins

116 weakly resemble to different subunits of the fusion complex of poxvirus, this

117 makes them appropriate candidates to analyse their function at the viral core

118 penetration to the cytoplasm.

119 ASFV protein E248R is a type II late structural protein required for viral membrane

120 fusion with limiting membrane of $\operatorname{LE}(33,35)$. It belongs to a class of myristoylated

121 proteins related to vaccinia virus (VACV) such as VACV L1R protein, which forms

122 part of the VACV fusion complex. L1R contains several disulfide bonds and it is

123 widely conserved in several families of Poxvirus $(36,37)$.

124 ASFV E199L is a transmembrane protein type I harboring disulfide bonds and

125 together with E248R, have been postulated to be necessary for membrane fusion

126 and core penetration to the cytoplasm (34). E199L resemble to three poxviral

127 fusion machinery subunits, named A16, G9 and A26. In absence of E199L, ASFV

128 infection drastically decreased and viral particles are retained into LE and

129 lysosomes.

130 In this report, we have shown that E248R and E199L, two candidate proteins

131 potentially involved in ASFV membrane process and located in the inner

132 membrane of the viral particle, interact with the LE integral membrane protein

133 NPC1. This novel finding supports an interaction of the internal membrane

134 proteins E248R and E199L with NPC1 C domain, thus bringing to the forefront

135 this cholesterol transporter as a crucial target to counteract the early steps of the

136 infection. 


\section{RESULTS}

\section{Cholesterol accumulation is critical for ASFV infection}

142 A first hint of the influence of cholesterol flux on ASFV infectivity was obtained by

143 using the U18666A chemical, a compound that blocks the NPC1 cholesterol

144 transporter. This compound reduces the cholesterol efflux from the endosome,

145 which remains retained inside these vesicles and its accumulation resulted in the

146 formation of large, dilated endosomes. We pre-treated Vero cells with increasing

147 concentrations of the U18666A drug and labelled cholesterol with Filipin antibody.

148 By these means, we correlated cholesterol retention with reductions in ASFV

149 infectivity at the same drug concentrations. Fig $1 \mathrm{~A}$ shows a characteristic

150 accumulation of cholesterol in large vesicle-like clusters around the perinuclear

151 area starting at a drug concentration of $0.5 \mu \mathrm{M}$. Likewise, Vero cells were pre-

152 treated with similar concentrations of U18666A drug and infected with ASFV

153 BPP30 GFP at a moi of $1 \mathrm{pfu} / \mathrm{ml}$ for $16 \mathrm{hpi}$. After this time, GFP expression was

154 tested in a plate reader. Importantly, infection started to be impaired at the same

155 concentrations that produced a cholesterol accumulation phenotype, suggesting

156 that there could be a correlation between both facts (Fig 1B).

158 Since ASFV is strongly dependent of acidic endosomal $\mathrm{pH}$ (11) we excluded the

159 possibility of a lysosomotropic effect of the $U$ drug by using the acid pH probe

160 Lysotracker. As positive controls, Vero cells were pre-treated with lysosomotropic

161 drugs Bafilomycin and $\mathrm{NH}_{4} \mathrm{Cl}$. Acidic vesicles were clearly stained in controls and

162 U18666A drug treated cells, while no signal from acidic vesicles was detected 
163 with Bafilomycin and $\mathrm{NH}_{4} \mathrm{Cl}$. This confirmed that the U18666A compound acted

164 specifically as an inhibitor of cholesterol export in this context and it has no action
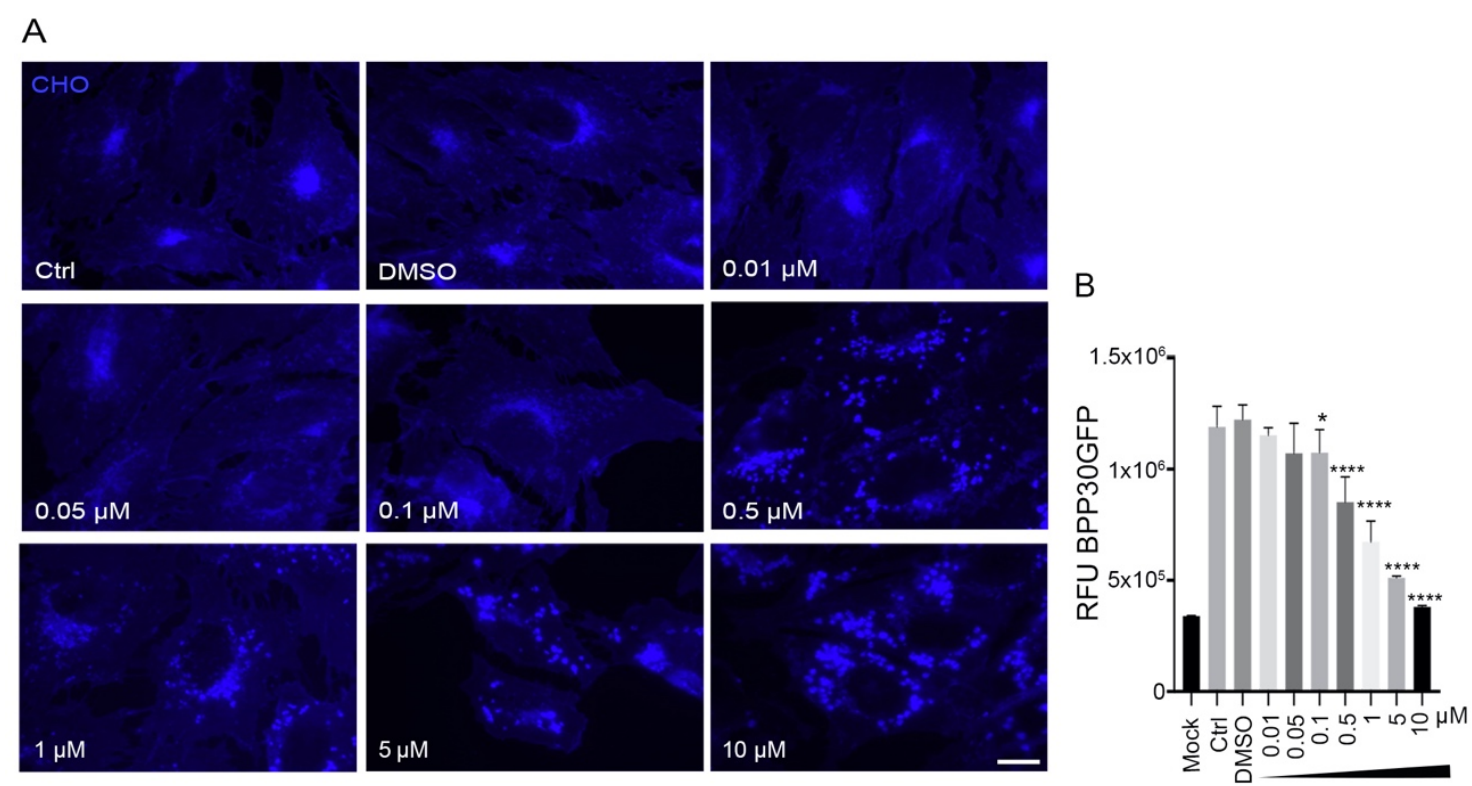

C
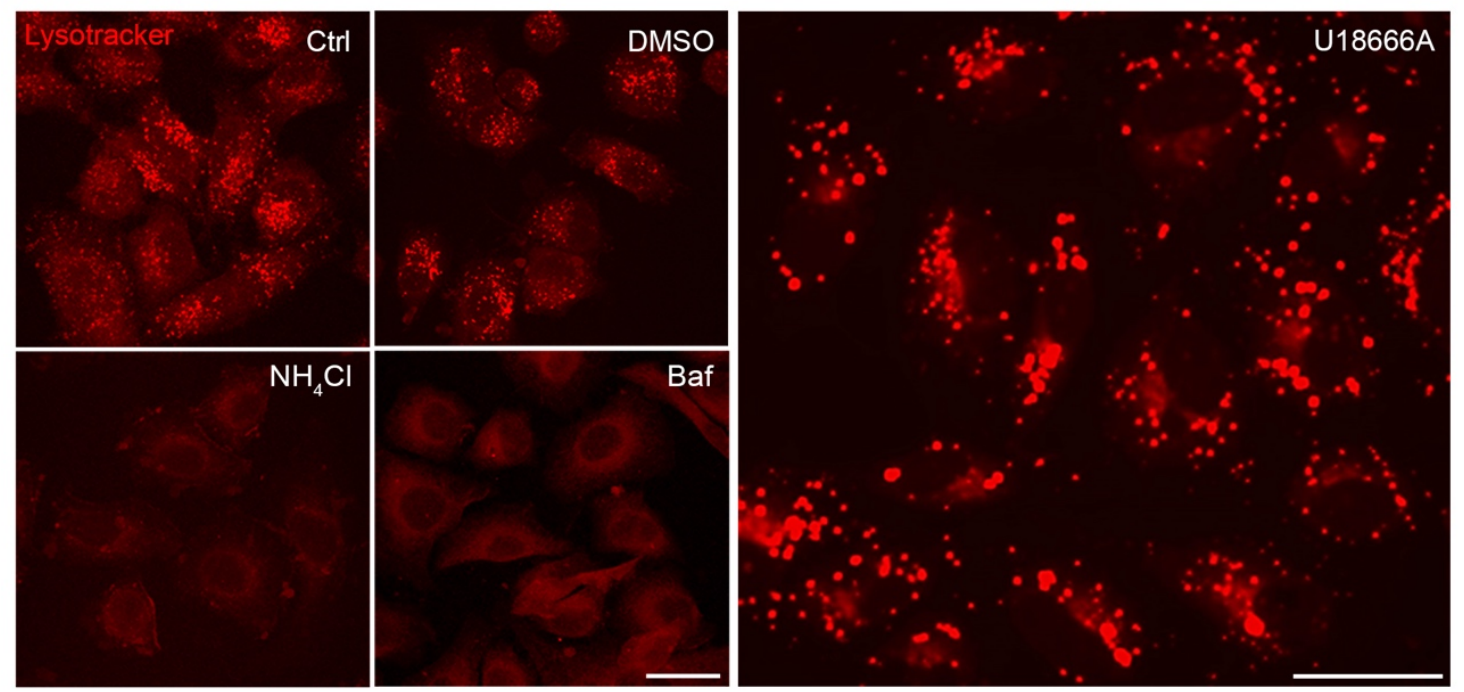

Fig 1. Cholesterol efflux blockade inhibition of ASFV infection. (A) Increasing concentrations of U18666A chemical compound altered the distribution of unesterified cholesterol (filipin; blue). Scale bar $10 \mu \mathrm{M}$. (B) Vero cells pre-treated

170 at increasing concentrations of $U$ drug were infected with recombinant ASFV

171 PP30GFP. After 16 hpi, GFP fluorescence intensity was measured by plaque assay. (C) Visualization of acidic vesicles with lysotracker (red) in cells treated 
173 with U18666A drug or controls, but not after lysosomotropic drugs $\mathrm{NH} 4 \mathrm{Cl}$ or

174 Bafilomycin treatment. Scale bar $25 \mu \mathrm{M}$.

175

176 The U18666A compound affects the cholesterol transporter function of NPC1

177 directly binding to the SSD of NPC1 resulting in the blockade of cholesterol efflux

178 out of endolysosomes, which also inhibits EBOV infection (19). This suggested

179 that the transporter role of NPC1 could be necessary for ASFV egress from the

180 LE to the cytosol as it was described for EBOV. This would ultimately affect viral

181 replication either if this occurs as a result of the lack of cholesterol efflux or

182 because of a mechanistic impairment. In EBOV infection, the viral glycoprotein

183 interacts directly with NPC1 to allow viral fusion and endosomal escape. Then,

184 we set out to investigate a possible similar mechanism mediated by analyzing the direct binding of an ASFV protein with NPC1.

\section{E248R and E199L sequence analysis}

188 As we mentioned above, E248R and E199L have been involved in membrane

189 fusion to finally conduct the naked viral core of the endocytosed virions from the

190 LE to the cytoplasm. These are late synthesized structural proteins highly conserved proteins among ASFV isolates (Fig 2A). Furthermore, both proteins resemble to different subunits of the entry/fusion multiprotein complex of poxvirus (S1 Fig).

196 E248R is a type II myristoylated transmembrane protein. It is composed of an N-

197 terminal portion of 199 amino acids, which contains four cysteine residues 198 oriented to the cytoplasm, a helical hydrophobic transmembrane domain of 21 
199 amino acids, and a 28 amino acid length extracellular region (35). E248R is the

200 final substrate of the ASFV-encoded redox system (38) and, as a consequence,

201 it could contain intramolecular disulfide bonds. We found that E248R yielded

$20216.2 \%$ identity and $30.7 \%$ similarity with VACV L1R fusion protein.

203 The distribution of cysteine residues in E248R (Fig 2C), compared to related

204 protein of VACV protein L1R would suggest that the two L1R disulfide bonds

205 between amino acids 28-62 and 122-156 could be also present in E248R (Fig.

206 2C and S1 Fig A).

207

$208 \mathrm{E} 199 \mathrm{~L}$ is a type I transmembrane protein with a N-terminal large cysteine

209 enriched portion oriented internally to the viral particle, and small transmembrane

210 and external (C-terminal) domains. E199L shares some degree of homology to

211 three cysteine enriched proteins belonging to the fusion machinery of VACV

212 named A16, A26 and G9, which also form disufide (39) bonds (Fig 2D and S1 Fig

213 B). 
A

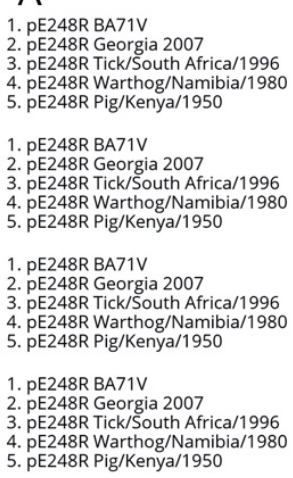

B

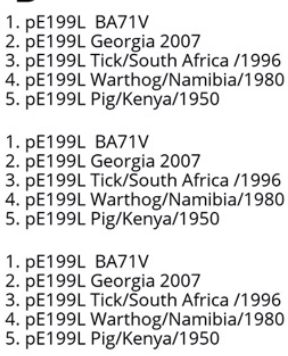

C

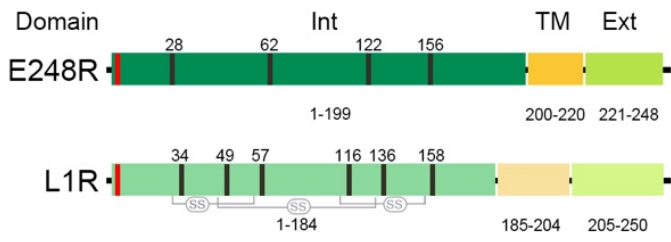

D
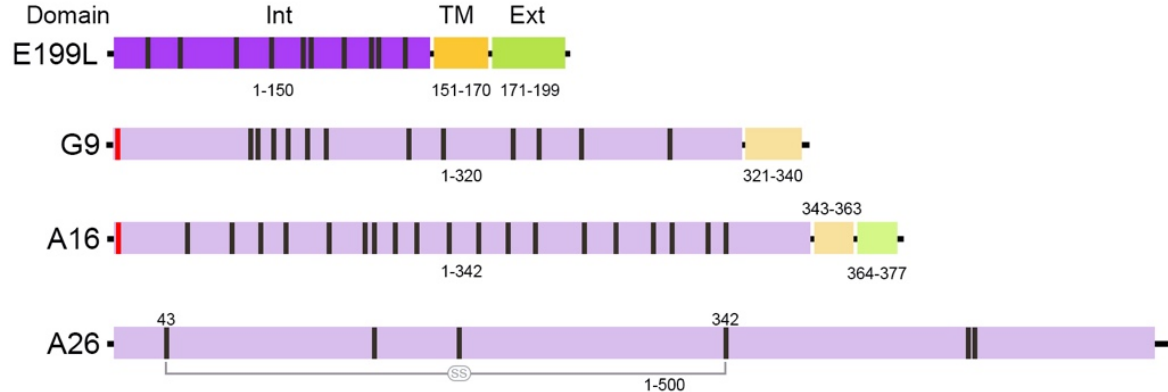

|Myristoylation ICysteine SS Disulfide bonds

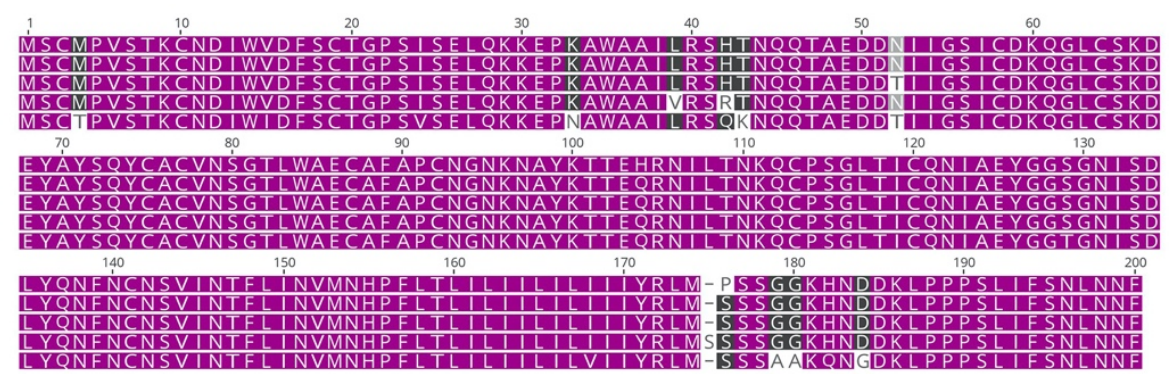
EYAYSOYCACVNSGTLWAECAFAPCNGNKNAYKT TEORN L TNKOCPSGLTICONIAEYGGSGNISD EYAYSOYCACVNSGTLWAECAFAPCNGNKNAYK TTEORNIL TNKOCPSGL TI CONIAEYGGSGN ISD EYYYSOYCACVNSGTLWAECAFAPCNGNKNAYKT TEQRN ILTNKQCPSGLTICQNIAEYGGTGNISD

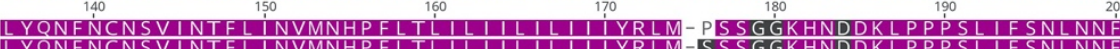
LYONFNCNSVINTFL INVMNHPFLTLIL ILIL IIIRL

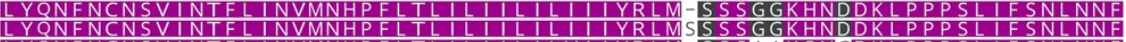

217 Fig 2. Comparison of ASFV E248R and E199L in isolates and with VACV

218 fusion proteins main domains. (A-B) Identical amino acids are marked green

219 for E248R or purple for E199L. Amino acids conserved 80\% are marked in dark

220 grey, those conserved between $60-80 \%$ in light grey. Finally, those preserved

221 less than $60 \%$ in white. (C-D) Structural comparison with VACV fusion proteins. 
222 Myristoylation signal is indicated in red, the three main domains: internal (Int),

223 transmembrane (TM) and external domains (Ext) represented in blocks. Amino

224 acid numbers are indicated. Black lines represent cysteines indicating the

225 positions above and the disulfide bonds in grey.

227 In order to determine a functional mechanism that would relate both viral proteins

228 with ASFV endosomal egress, we designed a protein-protein interaction experiment with NPC1, thus connecting cholesterol efflux in late endosomal vesicles with these proteins.

232 Interaction of E248R and E199L protein with NPC1

233 Cholesterol efflux mediated by NPC1 is crucial in ASFV infection and NPC1 is an

234 intracellular receptor for EBOV. Then, we tested the possibility of NPC1 being

235 involved in the fusion of viral and LE membranes, along with the viral proteins

236 E248R and E199L. These proteins located at the exposed inner membrane of

237 the virion after decapsidation are candidates for membrane fusion and viral core

238 penetration to the cytoplasm.

239 Schematics in Fig 3A depicts E248R domains and the deletion mutant

240 constructions designed for the experiment. E248R $\Delta$ Ext, lacking the external

241 protein domain, E248R $\triangle T M$ obtained by deletion of the transmembrane domain,

242 and E248R $\Delta$ Ext+TM, obtained by deletion of both transmembrane and external

243 domains. These deletions were constructed to clarify the relevance of each of the

244 ASFV protein E248R domains, exposing their role for binding the cellular 245 receptor.

246 First, to investigate the interaction of full-length E248R and E199L with NPC1-

247 Flag, both viral proteins were expressed as HA-fusions in HEK 293T cells, and 
248 independently co-expressed with NPC1-Flag. HEK 293T cells were selected due

249 to their high efficiency of transfection, being the cell line of choice for protein-

250 protein interaction studies of several viruses.

251 Protein expression of HA-E248R, mutants, HA-E199L and NPC1-Flag was

252 confirmed using western blot analysis (Fig 3B). NPC1-Flag or empty Flag were

253 then co-immunoprecipitated using Flag beads. After immunoprecipitation, both

254 input (cell lysate) and bound samples were analyzed by western blot.

255 Previous reports have shown the interaction between the primed form of GP

256 glycoprotein of EBOV and NPC1 cholesterol transporter to promote fusion with

257 the LE membrane (40). Since ASFV requires an intact cholesterol efflux from LE

258 (12) and traffics to the cytoplasm from LE compartment $(11,33)$ similarly to

259 EBOV, we analyse the putative implication of NPC1 in ASFV infection, through

260 an interaction with the suspected ASFV proteins involved in fusion with the LE,

261 E248R and E199L $(33,34)$.

262 As shown in Fig 3B, HA-E248R WT, HA-E248R mutants with TM domain, and

263 HA-E199L were detected in the immunoprecipitated pellet with Flag beads with

264 an HA antibody. NPC1 was also detected by using an antibody against Flag.

265 Conversely, an independent cellular protein fused to HA (HA-elF4E) used as a

266 negative control was not detected (Fig 3C), corroborating the interaction

267 specificity of E248R and E199L with NPC1. Altogether, these results demonstrate

268 that E248R interacts through its TM domain with NPC1, in fact E248R mutants

269 lacking TM domain shown no interaction with NPC1.

270 Simultaneously, we could also demonstrate VACV L1R direct binding to NPC1-

$271 \quad$ Flag (Fig 3D). 
A
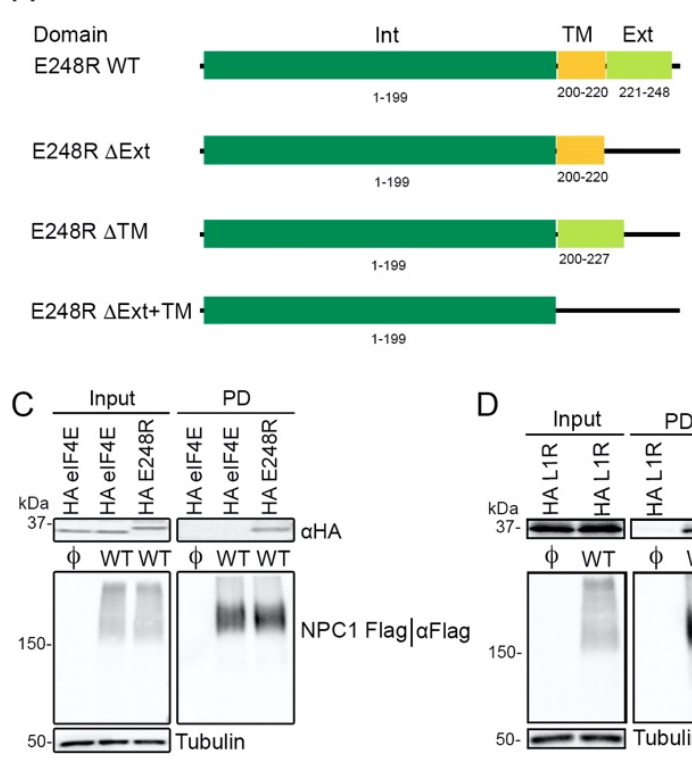

D
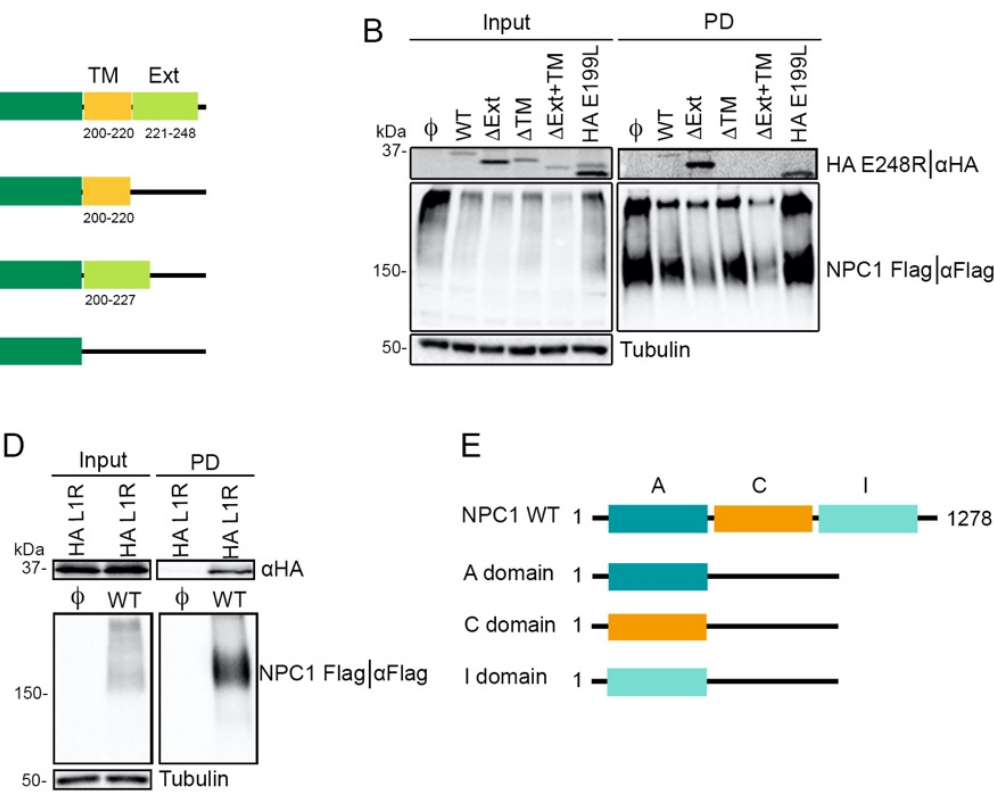

$\mathrm{E}$
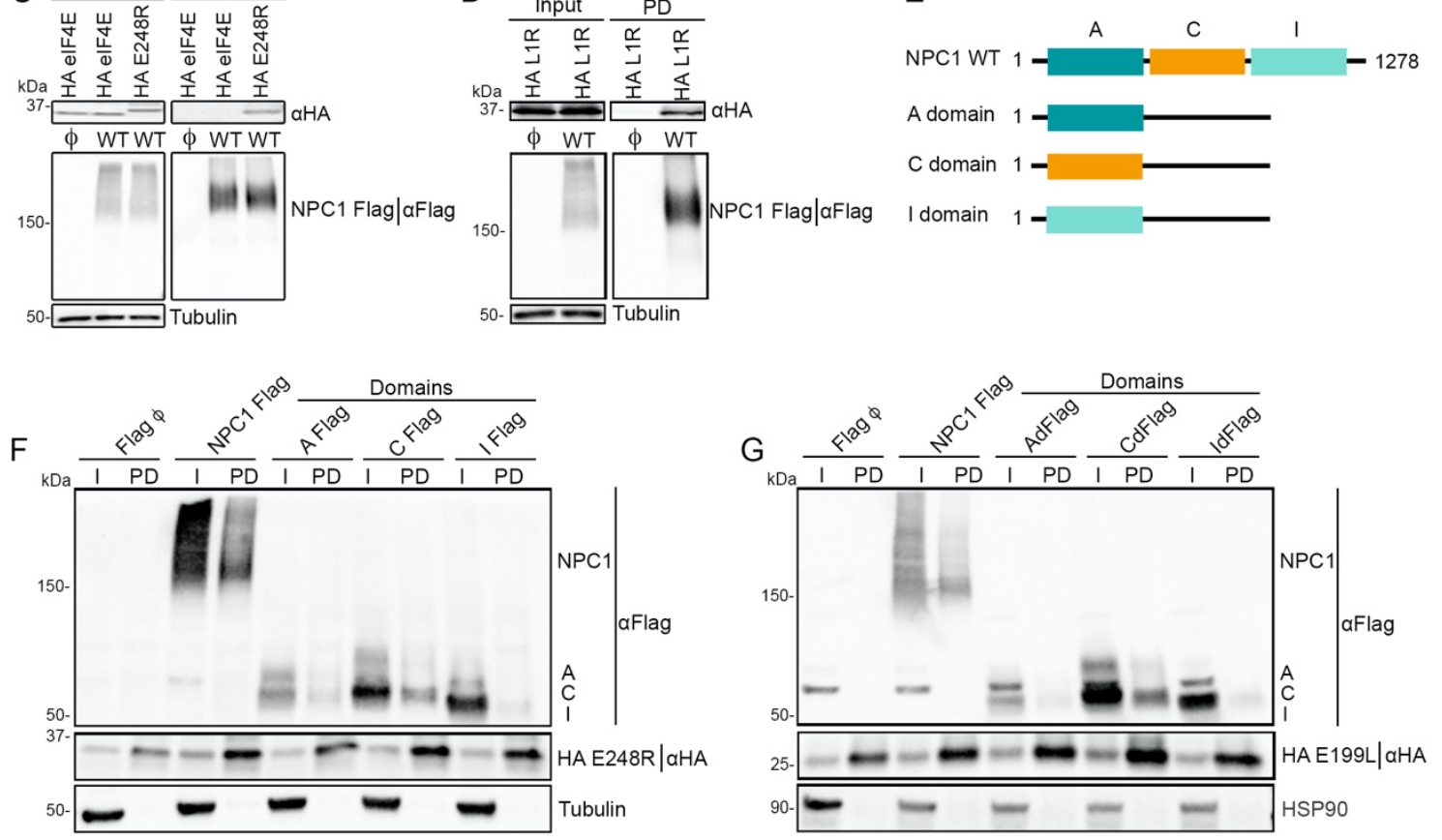

274 Fig 3. E248R and E199L interact with NPC1. (A) Schematic representation of

275 E248R WT protein and deletion mutant domains. (B) Detection of HA E248R WT,

276 HA E248R mutants and HA E199L WT co-expressed individually with NPC1 Flag

277 in 293T cells. HA and Flag fused proteins analyzed in the immunoprecipitation

278 assay ("PD" refers to pulled down sample) by western blot. (C) Flag pull-down experiments of 293T cells transiently co-expressing NPC1 Flag together with HA

280 E248R or HA elF4e as a negative control of the interaction analyzed by western

281 blot. (D) Flag pull-down experiments of 293T cells transiently co-expressing

282 NPC1 Flag together with HA L1R. (E) Schematic representation of NPC1 WT and 
284 with NPC1 Flag as a control for positive interaction, A Flag, C Flag or I Flag

285 construction ("PD" is the pull down sample). Interactions were detected by

286 reverse pulldown with Sepharose beads HA tagged were detected by western

287 blot. (G) Detection of co-expressed HA E199L (as a positive control of

288 interaction) with NPC1 Flag, A Flag, C Flag or I Flag construction ("PD” is the pull

289 down sample). Interactions were detected by reverse pulldown with Sepharose

290 beads HA tagged were detected by western blot. All immunoprecipitation assays

291 experiments were repeated three times to ensure reproducibility.

\section{E248R and E199L interact with NPC1 through C domain}

294 In order to identify the specific NPC1 domain involved in the interaction with

295 E248R, we designed three constructions expressing NPC1 individual domains A,

296 C, I fused to Flag (Fig 3E) (41). These domains were co-expressed individually

297 with HA E248R for 24 hours in 293 T cells. Co-immunoprecipitation with Flag

298 beads was undetermined, depicting an interaction with the three different NPC1

299 domains A, C and I, as analyzed by western blot (data not shown).

300 To further characterize the specific interaction between E248R with NPC1 and

301 the individual domains, the reciprocal co-immunoprecipitations (Co-IP or reverse

302 pull downs) against HA E248R were performed using protein G-beads and specific monoclonal antibodies against HA (Fig 3F). The expression of HA E248R

304 was confirmed using a HA antibody as expected but only NPC1 WT and C

305 domain were retrieved in the bound samples obtained from the Co-IP and 306 analyzed by WB.

307 A similar experiment was performed and resolved co-expressing HA E199L with 308 NPC1 WT and A, C and I domains fussed to Flag. We found a clear interaction 309 with NPC1 WT and C domain (Fig 3G). 
310 Thus, this result confirms the interaction between E248R via TM domain and

311 NPC1, through C domain of NPC1, similar to the EBOV GP interaction with NPC1

312 at the C domain. Also, E199L was found to interact with NPC1 via C domain.

\section{ASFV infection in NPC1 Knockout cells}

315 After confirming the interaction of both proteins with NPC1, CRISPR Cas9

316 technology was used to generate a knockout (KO) Vero cell line for NPC1

317 expression. The absence of NPC1 was confirmed from the pool of knocked cells

318 by western blot (WB), indirect immunofluorescence (IFI) and (S6 Fig A-B). Further

319 assays were performed to test the cellular phenotype that should be compatible

320 with the absence of NPC1 in the cells. The absence of NPC1 would result in a

321 cholesterol accumulation phenotype in dilated acidic vesicles, as we observed in

322 S6 Fig C. A similar cholesterol accumulation pattern than cells pre-treated with

323 U18666A compound was also observed in NPC1 KO pool of cells (S6 Fig D).

324 Moreover, since NPC1 acts as an intracellular receptor for filovirus, these KO

325 cells should be resistant to filovirus infection, as we verify by inhibition of VSV-

326 pseudotyped with GP EBOV Mayinga strain infection, confirming a functional 327 abrogation of NPC1 (S6 Fig E).

328 Susceptibility to ASFV infection remained similar between parental Vero WT cells 329 and a selected $\mathrm{LCV}_{2}$ clone (named as Empty), both were used as controls for

330 infection of NPC1 KO cells. After re-confirming the KO of NPC1 protein

331 expression by WB in several clones from the pool of KO cells, clone 14 (c14)

332 (named as NPC1 KO) was selected to perform further experiments. Also, we

333 stated the absence of NPC1 mRNA and the lack of protein expression by IFI (Fig 334 4A, 4B and 4C). 
A
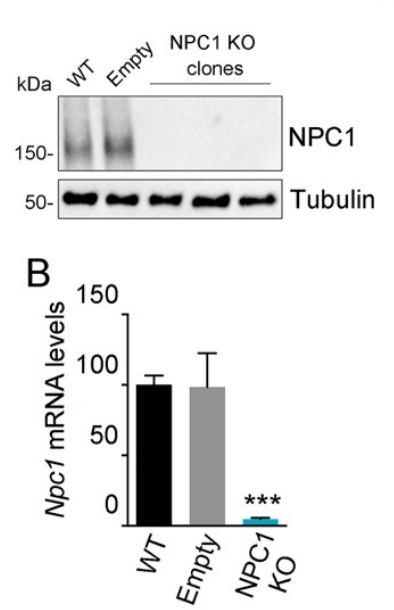

F
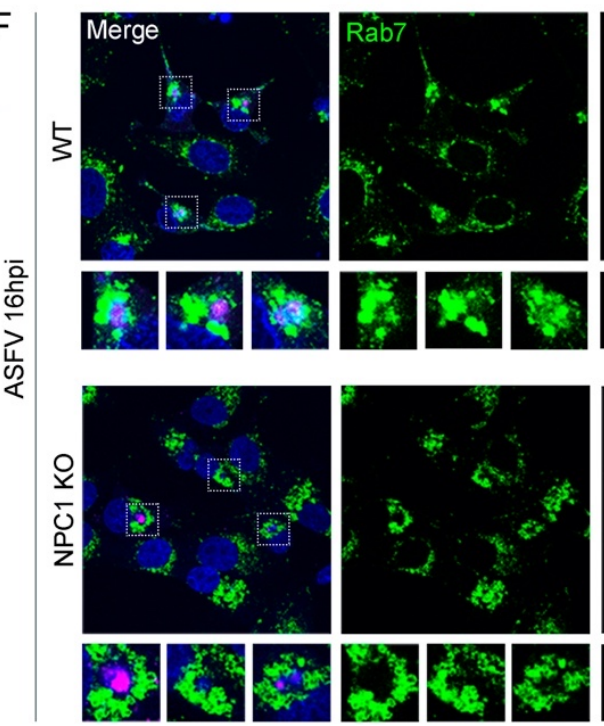

C
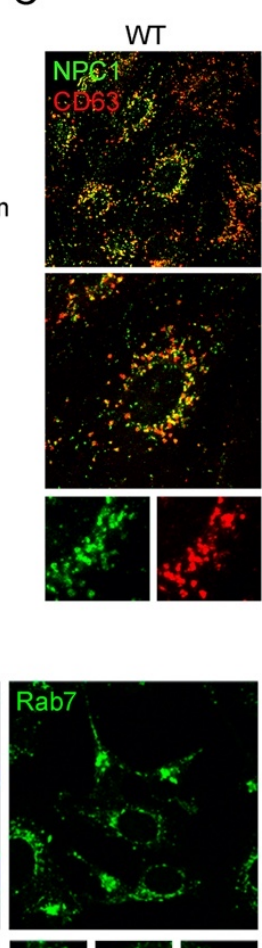
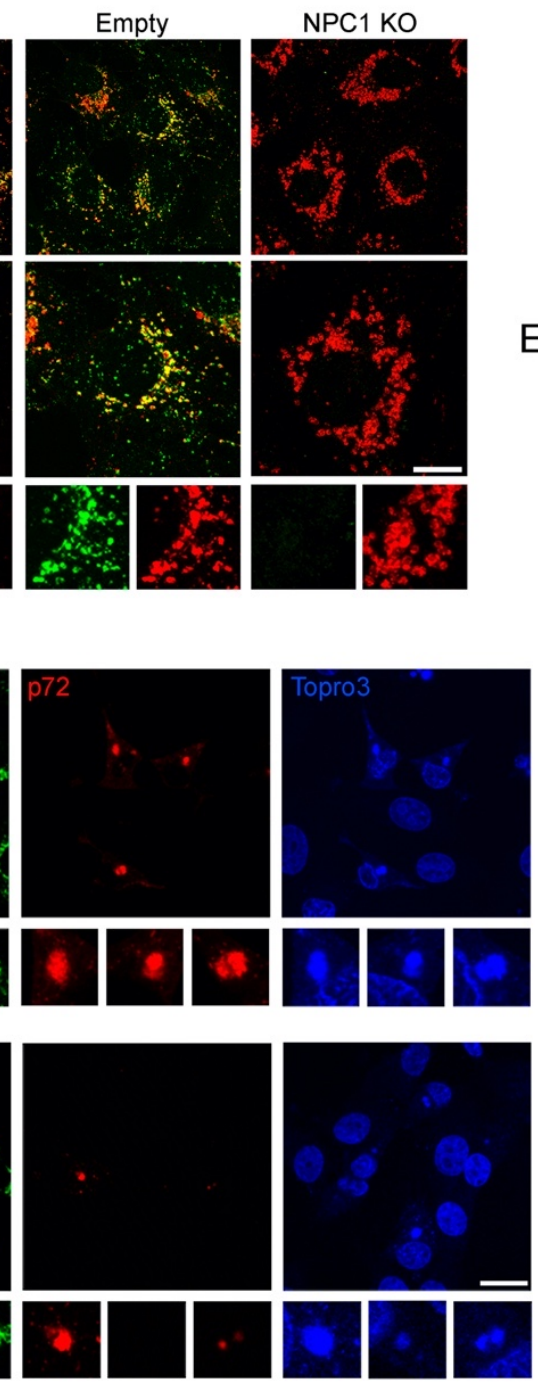

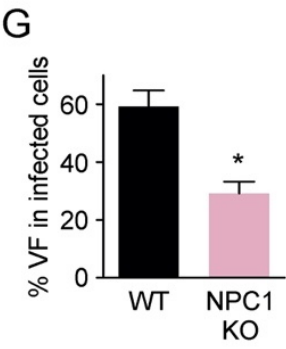

D
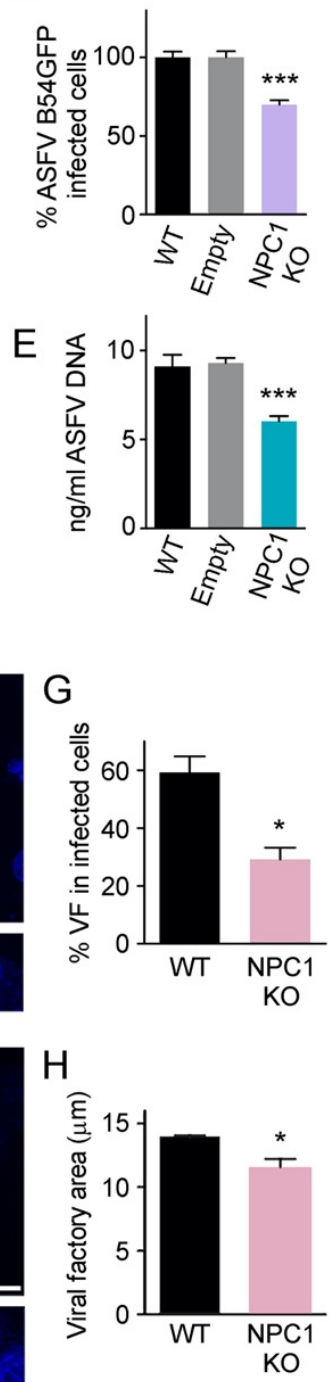

Fig 4. CRISPR KO NPC1 cells phenotype and ASFV infection. (A) Immunoblot

338 of NPC1 and tubulin (loading control) in WT, Empty and several clones of NPC1

339 KO cells. (B) Npc1 mRNA levels in WT, Empty and NPC1 KO Vero cells detected

340 by qPCR. C) Visualization NPC1 (green) in late endosomes (CD63 (red) in WT,

341 Empty and NPC1 KO cells. Scale bar: $25 \mu \mathrm{m}$. Zoom images are also shown. (D)

342 B54GFP infection percentages at 16h in WT, Empty and NPC1 KO Vero cells as

343 detected by flow cytometry. Percentages were normalized to values in WT cells.

344 (E) ASFV genome copy number in WT, empty and NPC1 KO ASFV infected cells 
analysed by real-time PCR. (F) Confocal images of Rab7 (green), ASFV p72 (red) and DNA (Topro3, blue) in WT and NPC1 KO cells. Scale bar: $20 \mu \mathrm{m}$. Zoom

347 images of ASFV viral factories (boxed regions) are also shown. (G) Percentage

348 of viral factories in WT and NPC1 $\mathrm{KO}$ in infected cells shown in $\mathrm{F} .(\mathrm{H})$

349 Quantification of the area of viral factories stained with ASFV p72 in WT and 350 NPC1 KO infected cells shown in F. (B, D, E, G and H). Graphs represent 351 mean \pm sem from three independent experiments. Statistically significant

352 differences are indicated by asterisks $\left({ }^{* * *} p<0.001,{ }^{* *} p<0.01,{ }^{*} p<0.05\right)$. As

353 controls we used both WT parental Vero cells and cells transduced with empty

354 vector. Once tested that infectivity and replication parameters were the same in both controls, we used Vero WT cells in following experiments.

Then, we analyzed the course of ASFV infection in these validated c14 NPC1 KO

358 Vero cells. A decrease below $40 \%$ of infectivity was detected by infection of

359 fluorescent recombinant ASFV, B54GFP by flow cytometry, which is a late protein

360 (Fig 4D). Likewise, ASFV replication was also affected according to decreasing

361 in number of ASFV genome copies (Fig 4E).

362 Importantly, similar results were obtained by using a different approach by 363 knocking down NPC1 with shRNA (S7 Fig).

364 The phenotype of NPC1KO Vero cells as expected showed an impediment for 365 cholesterol efflux resulting in the distribution of cholesterol within enlarged 366 endosomes in a closer perinuclear location in aggregates that was absent in 367 control Vero cells (Fig 5A, mock panel).

368 In order to compare the phenotype of mock and infected cells, we used an 369 antibody against ASFV major protein p72 that distributed in VF and accumulated 
370 together with viral DNA (Fig 4F, ASFV infected panel). The ASFV factories

371 depicted the characteristic compact appearance in large perinuclear individual

372 aggregates. However, in ASFV infected NPC1 KO Vero cells, the p72 stained

373 VFs were found in a smaller number of cells (Fig 4G) or were reduced in size in

374 a significant number of cells (Fig $4 \mathrm{H}$ ). In fact, there was less staining with antibody

375 against p72 than DNA staining with Topro3 in infected NPC1 KO Vero cells,

376 probably indicating a reduction in viral protein expression.

A
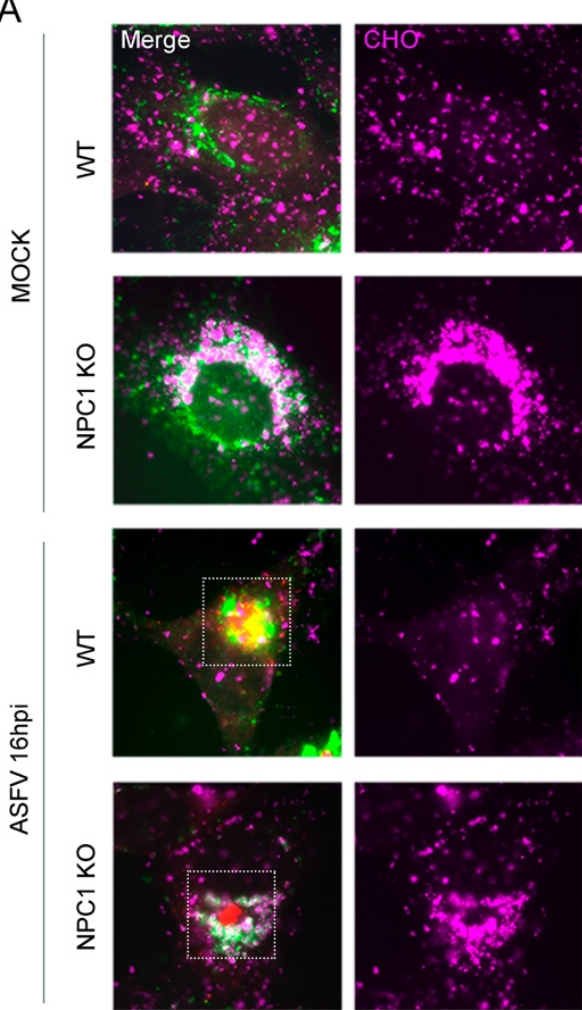
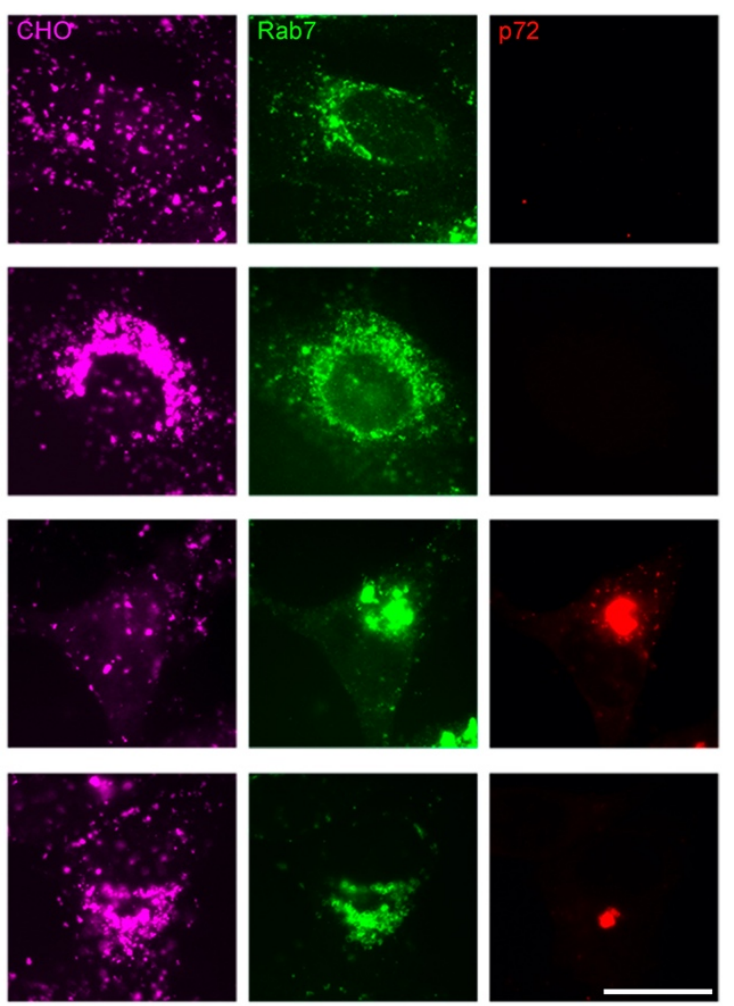

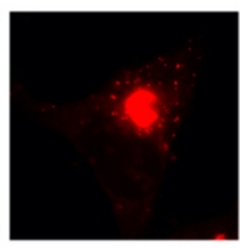

B
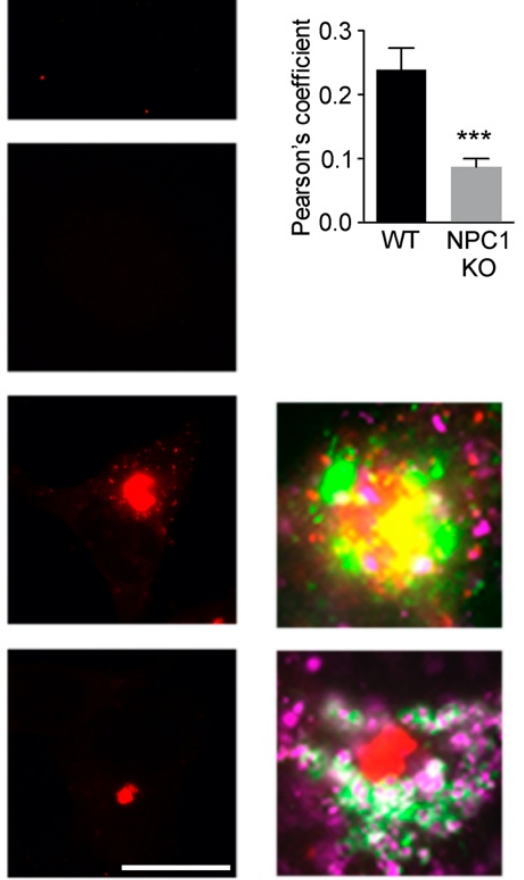

377

378

Fig 5. Colocalization of endosomes and viral protein at ASFV factories. (A) Confocal microscopy of merged images (left), cholesterol stained with filipin (CHO, magenta), Rab7 (green) and ASFV p72 (red) in mock or ASFV Ba71V infected Vero WT and NPC1 KO cells. A close apposition and colocalization of endosomes and viral proteins are seen at the factories. Scale bar: $20 \mu \mathrm{m}$. Zoom images of ASFV viral factories are also shown (right). (B) Quantification of Rab7 
385 and p72 colocalization in WT and NPC1 KO ASFV infected cells using ImageJ

386 software. Graph represents mean \pm sem of Pearson's coefficient of three

387 independent experiments. Statistically significant differences are indicated with

388 asterisks $\left({ }^{* * *} \mathrm{p}<0.001\right)$.

390 Cellular membranes have been postulated as platforms to support and sustain

391 the viral replication site constitution. According to (42), ASFV reorganizes and recruits endosomal membranes forming aggregates and clusters, interspersed and in close contact with the VF. We confirmed this close relationship in infected

394 Vero cells by IFI as a yellow colour was detected in the VFs in the merged image,

395 the viral protein p72 labelled in red, and late endosomal aggregates labelled in 396 green (Fig 5A). Surprisingly, membranes positive for Rab7 in green were also 397 detected around the VF in NPC1 KO cells, but those lacked the usual close 398 contact with the VF as detected by reduction of colocalization percentages, 399 probably suggesting a relevant function for NPC1 in supporting the architecture 400 of the VF (Fig 5B; ASFV 16h panel).

401 Hence, ASFV infection was altered in NPC1 KO Vero cells, especially important was the unusual architecture of the viral factories and the lower viral infectivity and viral replication with qPCR. 
A

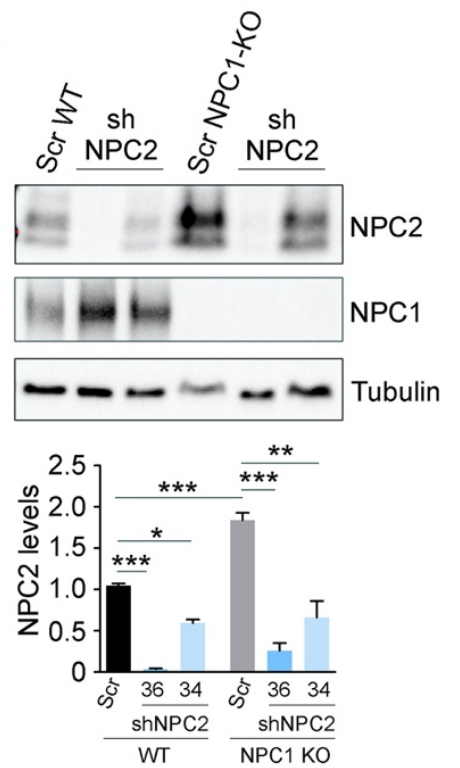

B

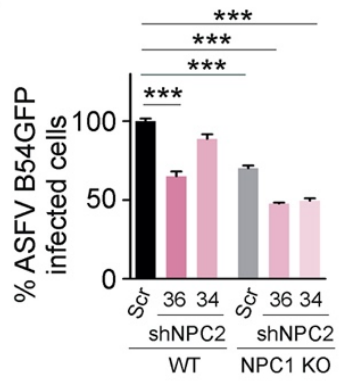

C
D
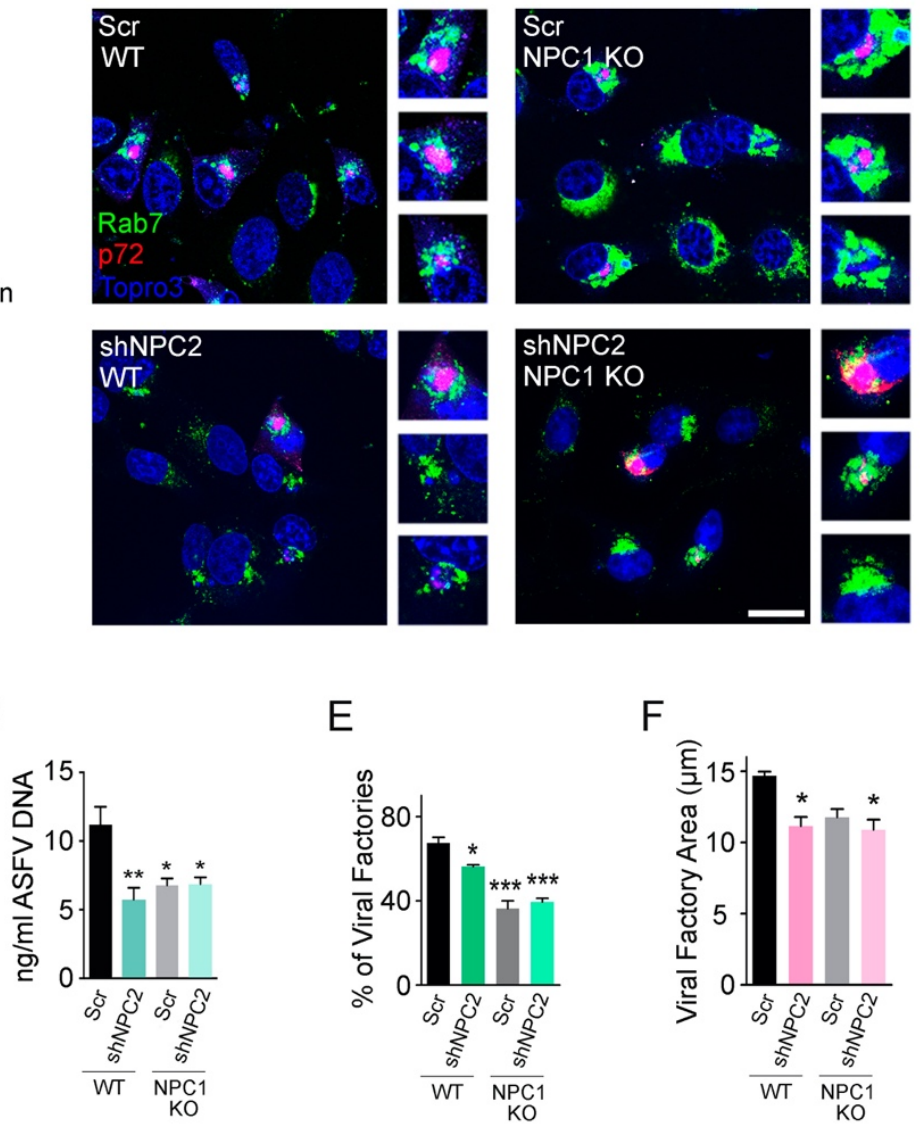

E

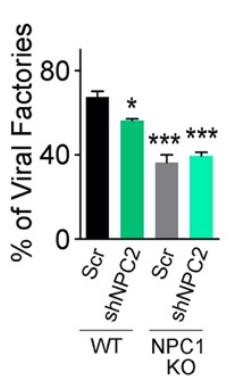

F

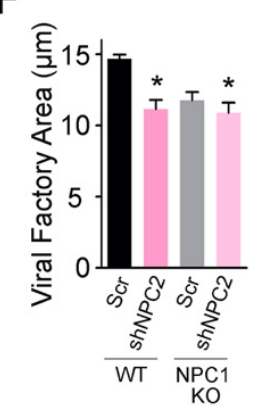

404

405

406

407

408

409

410

411

412

413

414 415 Confocal images of Rab7 (green), ASFV p72 (red) and DNA (Topro3, blue) in WT 416 and NPC1 KO cells transduced with Scr and shNPC2-36 lentiviral particles and

Fig 6. NPC2 downregulation reduced ASFV infectivity. (A) NPC1, NPC2 and tubulin (loading control) were detected by western blot in WT and NPC1-KO Vero cells transduced with Scr (scramble) and shNPC2 (34 and 36) lentiviral particles. Quantification of NPC1 and NPC2 bands were corrected to tubulin data and then normalized to WT-Scr values. (B) B54GFP infection percentages at $16 \mathrm{~h}$ in WT and NPC1 KO Vero cells transduced with Scr (scrambled) and shNPC2 (34 and 36) detected by flow cytometry. Percentages were normalized to values in ScrWT cells. (C) ASFV genome copy number in Scr and shNPC2-36 transduced WT and NPC1 KO cells, infected with ASFV and analysed by real-time PCR. (D) 
417 infected with ASFV. Scale bar: $20 \mu \mathrm{m}$. Zoom images of ASFV viral factories are

418 also shown. (E) Percentage of viral factories in cells shown in panel $E$. (F)

419 Quantification of the areas of viral factories stained with ASFV p72 in cells shown

420 in panel D. (A-C, E and F) Graphs represent mean \pm sem from three independent

421 experiments. Statistically significant differences are indicated by asterisks $\left({ }^{* * *} p<\right.$ $\left.0.001,{ }^{* *} p<0.01,{ }^{*} p<0.05\right)$.

423

424

425

\section{NPC2 knockdown reduces ASFV infection}

To gain mechanistic insight into the ASFV infection cycle, we studied the implication of NPC2 during this process. NPC2 is a single-domain luminal protein that works cooperatively with NPC1 to regulate the egress of endocytosed cholesterol from the late endosome $(13,16,43-45)$. To investigate whether NPC2 downregulation affects ASFV infection, we generated lentiviral particles encoding two different shRNA sequences (referred to as 34 and 36) for NPC2 silencing in WT and NPC1 KO Vero cells. The absence of NPC2 expression was confirmed by western blot (Fig 6A). Note that shNPC2-36 sharply decreased NPC2 levels both in WT and NPC1 KO Vero cells, while shNPC2-34 produced only a mild reduction of the target protein. Interestingly, NPC2 levels were significantly increased in NPC1 KO Vero cells as a consequence of NPC1 downregulation, however NPC2 downregulation did not affect NPC1 levels (Fig 6A).

NPC2 downregulation in WT cells showed a reduction of the infectivity measured with B54GFP virus compared to WT-Scr cells. Moreover, in Vero NPC1 KO cells, NPC2 downregulation potentiated the reduction of ASFV infectivity promoted by NPC1 abrogation (Fig 6B). ShNPC2-34 cells showed a discrete phenotype, possibly as a consequence of the mild reduction of NPC2 levels shown in Fig 6A. In this context, we found that ASFV replication was also affected by NPC2 
444 downregulation in WT and NPC1 KO cells, as observed in the quantification of

445 ASFV genome copies by qPCR (Fig 6C).

446 As we have shown above, NPC1 abrogation altered the distribution and shape of

447 late endosomes and the number and size of VFs in ASFV infected cells. To

448 address whether NPC2 could develop a similar phenotype, we stained Rab7 late

449 endosomes and ASFV p72 protein by immunofluorescence. Downregulation of

450 NPC2 did not induce such an altered phenotype in LE distribution in the factory

451 compared to NPC1 abrogation (Fig 6D); however, the number of ASFV VF and

452 their area were significantly decreased in shNPC2-36 infected cells compared to

453 control cells (Fig 6E and 6F) as occurred in NPC1 KO cells but at moderate levels.

454 Taken together, these results support that ASFV infection was affected by NPC2

455 downregulation in Vero cells, suggesting an important role of this protein during

456 ASFV infectivity.

\section{DISCUSSION}

460 ASFV infectious entry relies in the traffic of virions through the endocytic pathway

461 in an orchestrated multi step process depending on several molecular cues that

462 are characteristic of endosomes. Viral uncoating starts with an acidification463 dependent stage at the LE, a crucial compartment for the infection (11). Acidic

464 late endosomal environment is able to dissolve the viral capsids composed of 465 major capsid protein p72. Decapsidation allows the subsequent exposure of other 466 internal layers, necessary for further infection events. Beneath the capsid, once 467 the lipid inner viral membrane get exposed, it would eventually merge with the 468 limiting membrane of the LE. 
469 ASFV proteins potentially involved in fusion are located at the inner viral

470 membrane such as candidate fusion proteins E248R and E199L. These have

471 been postulated to be components of a hypothetical complex involved in ASFV

472 fusion, however, no interaction between them could be detected (34). These

473 proteins are highly conserved among ASFV isolates and they share some degree

474 of homology between E248R with VACV fusion protein L1R, and ASFV E199L

475 with VACV A16, A26 and G9 respectively (36). Moreover, E248R and E199L

476 belong to an ortholog cluster of genes from several viruses of the family of

477 nucleocytoplasmic large DNA viruses (NCLDV) (46), sharing conserved

478 structural motifs in several viruses of this broad family, which could indicate a

479 high functional importance of these proteins.

480 The ASFV fusion process would result in the egress of naked cores towards the 481 cytoplasm in an event that is strongly dependent on cholesterol efflux. Under 482 cholesterol efflux blockade, virions appear retained in endolysosomal vesicles.

483 We have previously shown that impairing cholesterol efflux results in secondary 484 cholesterol accumulation inside endosomes, which is able to inhibit ASFV 485 infection (12). In fact, we found here that both events were impacted at similar 486 concentrations of U18666A compound, which inhibits NPC1 receptor activity. The 487 required concentration of U18666A to inhibit ASFV infectivity was coincident with 488 the necessary concentration to evidence cholesterol retention inside endosomes 489 triggering cholesterol accumulation at the perinuclear area, suggesting that both 490 events are closely related. ASFV infection was blocked upon treatment with 491 U18666A drug, and virions were retained inside lipid-laden endosomes 492 suggesting an alteration at the fusion step. 
493 As a crucial cholesterol transporter protein, this observation also implied a

494 potential role for NPC1 in ASFV infection at the fusion step. Then, we set out to

495 study the viral fusion protein candidates, namely E248R and E199L. These are

496 highly conserved proteins among ASFV isolates located at the inner viral

497 membrane, which get exposed after decapsidation in the LE. Also, E248R has

498 been suggested to be involved in viral membrane fusion and core delivery but not

499 in viral disassembly inside the endosomes. Similarly, E199L is needed for viral

500 core penetration and seems not to be related with later processes, including

501 morphogenesis (33-35). Likewise, proteins belonging to the fusion complex of

502 VACV, a closely related virus, are crucial for DNA replication but not for

503 morphogenesis $(37,47)$.

504 In our sequence analysis of ASFV proteins E248R and E199L compared to 505 proteins belonging to the VACV multiprotein fusion complex, we found that

506 E248R yielded $16.2 \%$ identity and $30.7 \%$ similarity with VACV L1R fusion protein.

507 These interesting sequence similarities would suggest a parallelism between

508 ASFV and VACV fusion proteins and their functions among some of their 509 components.

510 Also, E248R and L1R have cysteines in similar positions in their sequence, which

511 indicate that the same cysteine oxidation mechanism that generates disulfide

512 bonds on the four cysteines in L1R, could also possibly occur in E248R. These

513 data together with the high sequence conservation of E248R, would suggest a

514 similar function to L1R. In fact, as above explained, we have found here a positive

515 interaction of VACV L1R with NPC1 similar to the ASFV fusion proteins that was

516 previously uncharacterized. 
518 We found a direct interaction between these candidate fusion proteins and

519 cholesterol transporter NPC1 by immunoprecipitation. E248R interacted with

520 NPC1 while no interaction was detected with an independent protein, elF4e,

521 included as a control in this study. Interestingly, E248R transmembrane domain

522 (TM) was found to be essential in this interaction, since TM domain lacking 523 mutants $(\triangle T M)$ were not pulled down by NPC1.

524 E248R-NPC1 interaction was re-tested and confirmed by reverse pull-down.

525 Besides, individual NPC1 domains A, C and I were included in the analysis,

526 importantly showing that the NPC1 C domain band (along with NPC1) was the

527 only domain detected in the E248R co-transfected bound pellet.

528 Collectively, these results demonstrate that E248R-NPC1 interaction would occur

529 via E248R TM domain with the NPC1 C domain, as it occurs at EBOV membrane

530 fusion and viral nucleic acid egress from the LE.

531 ASFV protein E199L was characterized as a virion protein expressed at late times

532 after infection and localized at the virus assembly sites (39). It is a

533 transmembrane protein type I located in the ASFV inner viral membrane. E199L

534 has also been reported to be a positive regulator of the NLRP3- (NLR Family

535 Pyrin Domain Containing 3) and AIM2- (Absent in melanoma 2) inflammasome

536 mediated inflammatory response (48). It has been also reported to be an

537 important antigenic protein in immunoassays (49).

538 In absence of E199L, ASFV infection drastically decrease and viral particles are

539 retained into LE and lysosomes, being necessary for viral membrane fusion and

540 core penetration (34). Interestingly, E199L resemble weakly in terms of sequence

541 identity to various poxvirus fusion subunits, named VACV proteins A16 (11.1\% 
542 identity and $20.87 \%$ similarity), A26 (10.2\% identity and $18.6 \%$ similarity) and G9

$543 \quad(12.2 \%$ identity and $25 \%$ similarity).

544 Remarkably, likewise E248R, we also found an interaction between E199L and

545 NPC1, confirmed by reverse pull-down. C domain co-transfected with E199L

546 were detected in the immunoprecipitated pellet.

547 Importantly, NPC1 has been demonstrated as an essential and common partner

548 for successful viral infections including distant viral families. NPC1 is a cholesterol

549 transporter at the endosomal membrane acting as a cellular receptor in the

550 cytoplasmic penetration of Ebola virus (EBOV; Filoviridae). Specifically, EBOV

551 protease-primed Glycoprotein (GP) (17) is able to interact with the C domain of

552 NPC1, triggering membrane fusion (50) and the absence of $C$ domain triggers

553 resistance to EBOV infection. The translocation of EBOV GP occurs

554 independently of cholesterol transporter function of NPC1 receptor $(19,51-53)$.

555 Hepatitis C virus (HCV) (Flaviviridae) requires a paralog of NPC1 named

556 Niemann Pick C1 like 1(NPC1L1) (27) on the surface of intestinal enterocytes

557 and human hepatocytes $(54,55)$, where it is responsible for homeostasis and

558 absorption of cholesterol. Also, a number of reports highlighted the relevance of

559 NPC1 in viral infections like Human immunodeficiency virus type-1 egress (HIV-

5601 1; Retroviridae). Moreover, the inhibition of NPC1 function by the chemical

561 compound U18666A was reported to inhibit the infection of Chikungunya

562 (Togaviridae), and other flavivirus including Zika Virus (ZIKV), West Nile Virus

563 (WNV), Yellow Fever Virus (YFV) and Dengue Virus (DENV), without any proven

564 interaction yet $(26,30,32)$. 
565 Recently, a positive interaction between NPC1 and SARS-CoV-2 Nucleoprotein

566 was reported (22). Consequently, NPC1 appears to be relevant in a variety of

567 RNA or DNA viral families.

568 Importantly, the U18666A compound is able to block the transport cellular

569 cholesterol from LE to several destinations by binding to the sterol sensing

570 domain (SSD) of NPC1 (19). LE is also the vesicle at which viral membrane fusion

571 occurs. LE is the site of cytoplasmic egress for late penetrating viruses such as

572 ASFV, and its integrity impacts severely viral replication and virion assembly (12).

573 Similarly, entry and replication of DENV, EBOV, ZIKV or CHIKV infections are

574 also profoundly affected under treatment with this drug $(32,40,56,57)$. In fact,

575 compounds specifically designed to interfere with NPC1 C domain, severely

576 impacted ASFV infectivity, supporting our observed interaction between NPC1 C

577 domain and both E248R and E199L (58).

578 Importantly, NPC1 abrogation completely inhibits EBOV infection, while ASFV as

579 a large complex virus could have evolved alternative cellular pathways, to

580 promote cholesterol efflux homeostasis. From this perspective, it is plausible that

581 due to its genome size, ASFV could have evolved alternative solutions within its

582 more than 150 ORFs, to overcome the abrogation of this cellular gene. However,

583 it is crucial to determine these potential cellular targets to design combined

584 therapies targeting the cellular components required for ASFV infection.

585 CRISPR KO NPC1 cell lines that inhibit EBOV infection, were partially resistant

586 to ASFV infection while NPC2 knockdown exerted an additive effect. Besides the

587 decrease in infectivity, we observed reduction of ASFV replication in these KO

588 cells. The number of cells harboring viral factories decreased and these

589 presented altered morphology, smaller size and less contacts between the 
590 endosomal membranes as supporting elements of the viral factory. Similar to

591 human cytomegalovirus (HCMV; a DNA virus), the constitution of the ASFV viral

592 factory (42), replication and assembly of new progeny occurs in close relationship

593 with lipid-rich cellular membranes. Then, it is possible that an intact cellular

594 cholesterol flux might be also required to facilitate ASFV replication after viral

595 entry.

596

597 In summary, ASFV cholesterol efflux blockade in the presence of the U18666A

598 compound drives virion retention inside cholesterol-laden endosomes, inhibiting

599 infection by altering NPC1 function. Our data support a novel NPC1 function to

600 facilitate ASFV membrane fusion and core penetration, according to the direct

601 interaction of ASFV proteins E248R and E199L with this endosomal receptor.

602 Importantly, these ASFV proteins are highly conserved among ASFV isolates and

603 share homology with components of the VACV entry/fusion complex. Binding

604 occurs at the late endosome between the transmembrane domain of both fusion

605 proteins with NPC1 domain C similar to EBOV.

606 These findings provide further knowledge of the molecular interactions underlying

607 viral fusion and pave the way to the clarification of the function of NPC1 and

608 NPC2 in fusion and early formation of the replication sites for this and other

609 relevant virus models that will be subject of further studies.

610

611 


\section{MATERIAL AND METHODS}

613

\section{Cell lines}

615 Vero and Human embryonic kidney cells (HEK293T or 293T) were grown at 37

$616{ }^{\circ} \mathrm{C}, 5 \% \mathrm{CO}_{2}$ atmosphere culture in complete Dulbecco's Modified Eagle Medium

617 (DMEM) containing 5 or $10 \%$ heat-inactivated fetal bovine serum (FBS)

618 respectively, 1\% penicillin-streptomycin (P/S) and 1\% Glutamax (Gibco,

619 Gaithersburg, MD, USA).

620

\section{Viruses and Infection}

622 We used the cell culture-adapted and non-pathogenic ASFV isolate Ba71V (59),

623 the recombinant virus from parental Ba71V, expressing GFP as a fusion protein 624 of p54 protein (B54GFP) (60) isolates or the recombinant Ba71V-30GFP 625 (BPP30GFP) (61). ASFV viral stocks were propagated and titrated by plaque 626 assay in Vero cells, as previously described (59). When using the recombinant 627 virus B54GFP, green fluorescent plaques were observed 4 days after infection 628 under the fluorescence microscope. For immunofluorescence, ASFV stocks were partially purified using a sucrose cushion (40\%) in PBS at $68,000 \times \mathrm{g}$ for $50 \mathrm{~min}$

630 at $4^{\circ} \mathrm{C}$ and were further used at a multiplicity of infection (moi) of 1 unless 631 otherwise indicated.

632

633 U18666A chemical compound treatment

634 U18666A (Sigma) is a chemical compound described as a direct target NPC1

635 function by binding NPC1 sterol sensing domain (19). It has been widely used to 636 interfere the endosomal cholesterol efflux, mimicking the phenotype observed in 
637 Niemann-Pick type C disease $(62,63)$. Cytotoxicity in Vero cells was previously

638 tested using CellTiter 96 (Promega). Vero cells were pre-treated with DMSO or

639 increasing concentrations of U18666A drug ranging from 0.01- $10 \mu \mathrm{M}, 16 \mathrm{~h}$ before

640 infection without washing leaving compound during the infection.

641

\section{$642 \quad$ Plasmids and constructs}

643 Plasmid pKH3 3xHA was purchased from Addgene (ref. 12555) and used to clone

644 in frame with the HA tag in the N-terminus the viral proteins E248R and E199L 645 into the Bam $\mathrm{HI}$ and Eco RI restriction sites.

646 E248R deletion mutant proteins of distinct domains ( $\Delta$ constructs) were 647 generated from E248R WT plasmid by site direct mutagenesis using the Q5 648 mutagenesis kit (New England Biolabs) as follows. E248R $\Delta$ Ext construct was 649 obtained by deletion of the outer protein domain (from nt 661 to nt 747); E248R $650 \Delta \mathrm{TM}$ was obtained by deletion of the transmembrane domain (from nt 601 to $\mathrm{nt}$ $651660)$; and E248R $\Delta$ Ext+TM was obtained by deletion of both TM and Ext domains 652 (from nt 601 to $\mathrm{nt} 747$ ).

653 Sus scrofa NPC1 WT-linker was generated by gene synthesis (GeneArt, 654 ThermoFisher) with a gly-gly-gly-ser linker followed by $3 x$ Flags at $C$ terminus 655 end as a tag into pCDNA 3.1+.

656 Plasmids expressing a single domain (,$C$ or I domain) from full length NPC1 657 were subcloned as N-terminal 3xFlag fusions into a pCDNA 3.1+ containing the 658 NPC1 signal peptide, the transmembrane domain and the tail. A gly-gly-gly-ser 659 linker was located before the 3xFlag tag. 
662 For pulldowns, 293T cells at $80 \%$ confluence were plated 24 hours before

663 transfection in P60 dishes. Prior transfection, the media was changed to DMEM

664 complete media at $2 \%$ FCS. Then, the different NPC1 constructs (NPC1, NPC1

665 A domain, NPC1 C domain and NPC1 I domain) and the viral protein constructs

666 were co-transfected using Lipofectamine 2000 (Invitrogen) following the

667 manufacture instructions.

668

669 Pull downs

670 293T cells were plated in P60 plates the day before transfection and co-

671 transfected with corresponding pCDNA3.1+ 3XFlag and pKH3 3xHA tagged

672 constructions during 24 hours. Then, cells were lysed in $1 \mathrm{ml}$ of ice-chilled lysis

673 buffer consisting in $50 \mathrm{mM}$ Tris, pH 7.4, $150 \mathrm{mM} \mathrm{NaCl}, 5 \mathrm{mM}$ EDTA, 5\% glycerol,

$6741 \%$ Triton X-100 and a protease inhibitor cocktail (complete mini-EDTA free,

675 Sigma), and then, incubated 30 minutes (min) under rotation at $4^{\circ} \mathrm{C}$. After, lysates

676 were centrifuged at $15000 \mathrm{rpm}$ for $30 \mathrm{~min}$ at $4^{\circ} \mathrm{C}$ and incubated with Flag coated

677 beads for 3 hours (h) and $30 \mathrm{~min}$ at $4^{\circ} \mathrm{C}$ under rotation. Finally, the lysates were

678 washed five times with wash buffer (50 mM Tris, $\mathrm{pH} 7.4,150 \mathrm{mM} \mathrm{NaCl}, 5 \mathrm{mM}$

679 EDTA, 5\% glycerol, and $0.1 \%$ Triton $\mathrm{X}-100)$ and bound proteins were eluted in

$68060 \mu \mathrm{l}$ of Laemmli buffer and boiled at $100^{\circ} \mathrm{C}$. Input and pulled down samples were

681 analyzed by western blot.

682

683 Reverse Pull down for HA viral tagged proteins

684 Twenty-four hours post-transfection, the cell pellet was resuspended in $200 \mu$ l of

685 Iysis buffer (10mM Tris/Cl pH 7.5; 150mM NaCl; 0.5mM EDTA; 0.5\%NP40) and

686 then incubated for 30 minutes on ice. The lysate was then clarified by 
687 centrifugation at $14000 \times \mathrm{g}$ and diluted five-fold with dilution buffer $(10 \mathrm{mM} \mathrm{Tris} / \mathrm{Cl}$ $688 \mathrm{pH} 7.5 ; 150 \mathrm{mM} \mathrm{NaCl} ; 0.5 \mathrm{mM}$ EDTA) up to $1 \mathrm{ml}$.

689 The HA pull down was performed using $50 \mu$ l of the Immobilized Recombinant

690 Protein G Resin (Generon) and specific antibodies against HA (Invitrogen).

691 In order to perform the pull down, a pre-clearing step was done by incubating the

692 diluted cell lysate with $50 \mu$ l of the Immobilized Recombinant Protein G Resin

693 (Generon) on a rotator for 2 hours at $4^{\circ} \mathrm{C}$ followed by centrifugation at $2500 \mathrm{~g}$ for

6942 minutes to collect the cell lysate from the beads. Then $2.5 \mu \mathrm{g}$ of the primary 695 antibody was added into the cell lysate and incubated at $4{ }^{\circ} \mathrm{C}$ on a rotator for 696 another two hours. Then, 50 ul of the protein $\mathrm{G}$ resin (Generon) were equilibrated 697 with ice-cold dilution buffer and then incubated at $4^{\circ} \mathrm{C}$ on a rotator with diluted 698 cell lysate containing the antibody overnight at $4^{\circ} \mathrm{C}$ on a rotator, followed by 699 centrifugation at $2500 \mathrm{~g}$ for 2 minutes to remove non-bounds fractions.

700 The bead pellet was washed once with wash buffer (10mM Tris/Cl pH $7.5 ; 150 \mathrm{mM}$

$701 \mathrm{NaCl} ; 0.5 \mathrm{mM}$ EDTA) and a second time with the wash buffer containing a higher 702 salt concentration (10mM Tris/Cl pH 7.5; 300mM NaCl; 0.5mM EDTA). After removal of the wash buffer, beads were resuspended in $100 \mu$ l of Sample

704 Buffer, Laemmli $2 \mathrm{X}$ Concentrate (Sigma Aldrich) and boiled at $95^{\circ} \mathrm{C}$ for ten min 705 to elute the bound proteins. Buffers used for Immunoprecipitations were all 706 supplemented with HaltTM Protease Inhibitor Cocktail EDTA-Free (Thermo 707 Fisher Scientific).

Western blot analysis and antibodies

710 Samples from pulldowns were eluted, boiled in Laemmli buffer and resolved by 711 SDS-PAGE in $7 \%, 12 \%$ or $15 \%$ acrylamide-bisacrylamide gels or in Mini- 
712 PROTEAN TGX Gels (Bio-Rad) then, the gels were transferred to a nitrocellulose

713 membrane (Bio-Rad) using the Trans-Blot Turbo Transfect Pack (Bio-Rad) and

714 the Trans-Blot Turbo system (Bio-Rad) and detected with corresponding

715 antibodies anti-Flag (Sigma), anti-HA (Invitrogen), and anti-Tubulin (Sigma) or

716 HSP90 (Palex) as load controls in Western blot (WB) analysis. As a secondary

717 antibody, anti-mouse IgG (GE Healthcare, Chicago, IL, USA) or anti-rabbit IgG

718 (Bio-Rad) conjugated to horseradish peroxidase was used at a 1:5000 dilution.

719 Finally, bands obtained after development with ECL reagent were detected on a

720 Molecular Imager Chemidoc XRSplus imaging system. Bands were quantified by

721 densitometry and data normalized to control values using Image lab software

722 (Bio-Rad).

723

724 Generation of Vero NPC1-knockout by CRISPR/Cas9 technology

725 We selected the small guide RNA1 (sgRNA): NPC1 KO Fwd: 5'726 CACCGCAAACTTGTATCATTCAGAG-3' and NPC1 KO Rvs: 5'

727 AAACCTCTGAATGATACAAGTTTGC-3', among 4 candidate target sequences

728 at the genomic region of Vero NPC1 region, designed with the tool Deskgen (64).

729 The sgRNA was cloned into LentiCRISPRv2 vector according to the

730 manufacturer indications (GeCKO Lentiviral Crispr toolbox, ZhangLab) and along

731 with plasmids PsPAX2 and VSVg were transfected in 293T cells (as previously

732 described) cultured the day before in p100 plates to generate the lentiviral

733 particles. Similarly, lentiviral particles were also generated with an empty

734 LentiCRISPRv2 as a control (selected clone was named as Empty). Lentiviral

735 particles were harvested $72 \mathrm{~h}$ after transfection and centrifugated at $4000 \mathrm{rpm}$ for

7363 min to eliminate cellular debris. 
737 The cleared Lentiviral containing medium was transduced in pre-treated Vero cell

738 with Polibrene $(8 \mathrm{ug} / \mathrm{ml})$ for $5 \mathrm{~min}$ and plated the day before in MW6 plates.

739 After 72 hours post transduction, the medium was shifted to DMEM $10 \%$ FBS

740 containing puromycin $(20 \mathrm{ug} / \mathrm{ml})$ to select transduced cells. Finally, Vero cells

741 transduced with lentivirus expressing sgRNA1 were selected after checking the

742 absence of NPC1 by WB and indirect immunofluorescence (IFI) with an antibody against NPC1 (Abcam). We also were able to confirm the typical cholesterol accumulation into laden late endosomes located in a perinuclear position, a usual phenotypic change produced under NPC1 depletion conditions that mimics the

Niemann Pick type C disease in CRISPR KO cells. Then, we perform a limited

747 dilution to plate individual clones on $96 \mathrm{MW}$ plate. After clonal expansion during 3 weeks, they were newly seeded in MW6 plates, expanded and finally checked and selected by the absence of NPC1 expression by WB compared to controls, and sequenced with primers

Fwd: 5

GCGCGCCTAGAAATTTAGAAGTCGTT-3'. Of the 31 clones examined, we selected c14, c19, c30 and the c14 was used for the experiments (named as NPC1 KO).

\section{Effect of NPC1 KO on infectivities of pseudotyped VSVs.}

757 We further confirmed NPC1 KO cells status by a functional assay testing their

758 resistance to Ebola virus infection using pseudovirions of vesicular stomatitis virus with the EBOV glycoprotein. Infection of control Vero parental cells and Vero NPC1 KO cell lines with recombinant VSV (rVSVs) bearing EBOV glycoprotein

761 (GP) of Mayinga strain or VSV-G were assayed. After $24 \mathrm{~h}$ post inoculation, cells 
762 were lysed, and luciferase expression measured in a luminometer as Relative

763 Light Units (RLU). The percentages of infectivity were determined by setting the

764 number of RLU in Vero cells to $100 \%$ for each envelope.

766 Flow cytometry analysis

767 Detection of ASFV infected cells was performed by flow cytometry. Vero cells 768 were infected with recombinant ASFV B54GFP at a moi of 1 pfu/cell for $16 \mathrm{~h}$.

769 Cells were washed with PBS, harvested by trypsinization, and then washed and

770 collected with flow cytometry buffer (PBS, $0.01 \%$ sodium azide, and $0.1 \%$ bovine

771 serum albumin). In order to determine the percentage of infected cells per

772 condition, 10,000 cells/time point were scored and analyzed in a FACS Canto II

773 flow cytometer (BDSciences). Infected cell percentages obtained were

774 normalized to values found in control samples.

775

776 Detection and quantitation of the ASFV genome

777 The quantitation of the number of copies of the ASFV genome was achieved by

778 quantitative real-time PCR (qPCR). DNA from Vero cells infected with ASFV at a

779 moi of 1 pfu/cell for $16 \mathrm{hpi}$, was purified using the DNAeasy blood and tissue kit

780 (Qiagen) following the manufacturer's protocol. DNA concentration was

781 measured using a Nanodrop spectrophotometer. The qPCR assay used

782 fluorescent hybridization probes to amplify a region of the p72 viral gene, as

783 described previously (65). The amplification mixture was $200 \mathrm{ng}$ of DNA template

784 added to a final reaction mixture of $20 \mu$ l containing 50 pmol sense primers, 50

785 pmol anti-sense primer, 5 pmol of probe and $10 \mu$ of Premix Ex Taq (2x) (Takara).

786 Positive amplification controls were DNA purified from ASFV virions at different 
787 concentrations used as standards. Each sample was included in triplicates and

788 values were normalized to standard positive controls. Reactions were performed

789 using the ABI 7500 Fast Real-Time PCR System (Applied Biosystems) with the

790 following parameters: $94{ }^{\circ} \mathrm{C} 10 \mathrm{~min}$ and 45 cycles of $94{ }^{\circ} \mathrm{C}$ for $10 \mathrm{~s}$ and $58{ }^{\circ} \mathrm{C}$ for

$79160 \mathrm{~s}$.

792 For quantitation of Npc1 mRNA levels, RNA was extracted from Vero cells using

793 RNeasy RNA extraction kit (QIAgen) according to the manufacturer's protocol.

794 For retrotranscription QuantiTect Reverse Transcription kit (QIAgen) was used to

795 synthesize cDNA, also following the manufacturer's protocol. $250 \mathrm{ng}$ of cDNA

796 were used as the template for real- time PCR using QUANTITECT SYBR GREEN

797 PCR KIT (QIAgen). Reactions were performed using the ABI 7500 Fast Real-

798 Time PCR System (Applied Biosystems). Expression of Npc1 gene was

799 normalized to an internal control (18S ribosome subunit), and these values were

800 then normalized to the value of control cells to yield the fold reduction. The

801 following primers were used: Npc1_fwd (5' GTGTGGTGCTACAGAAAACGG 3'),

802 Npc1_rev (5' AAATGCTGCACTGACAGGGT 3'). 18S ribosome subunit primers

803 provided in QuantiTect Primer Assay (Quiagen) were used as internal control.

804

805 Silencing shRNA

806 Lentiviral vectors containing shRNAs to interfere Npc1 (shNPC1) and Npc2

807 (shNPC2) were purchased from Merk (Darmstadt, Germany). Two different

808 sequences were transduced for shNPC1 and shNPC2: 
811 CCGGAGAGGTACAATTGCGAATATTCTCGAGAATATTCGCAATTGTACCTC

812 TTTTTTTG (TRCN0000421158, sh NPC1 \#58)

813 CCGGCGGTTCTGTGGATGGAGTTATCTCGAGATAACTCCATCCACAGAAC

814 CGTTTTTG (TRCN0000293234, shNPC2 \#34) and

815 CCGGGCTGAGCAAAGGACAGTCTTACTCGAGTAAGACTGTCCTTTGCTCA

816 GCTTTTTG (TRCN0000293236, shNPC2 \#36). A TRC2 pLKO.5-puro Non-

817 Target shRNA was used as control.

818 Lentiviral suspensions were prepared in HEK293T. HEK293T were transfected

819 with lentiviral and packaging vectors (psPAX2 and p-CMV-VSV-G) using

820 Lipofectamine $2000 \AA$ reagent and OPTI-MEM media (ThermoFisher Scientific,

821 Massachusetts, EEUU) for $4 \mathrm{~h}$ following the manufacturer instructions. Medium

822 was then changed to Iscove's Modified Dulbecco's Medium (IMDM)

823 complemented with 10\% FBS, 1\% penicillin-streptomycin (P/S) and 1\% Glutamax

824 (Gibco, Gaithersburg, MD, USA). Supernatant containing viral particles was

825 collected after 24 and $48 \mathrm{~h}$, centrifugated at $3000 \mathrm{rpm}$ for $5 \mathrm{~min}$ to eliminate

826 cellular debris and filtered using a $0.45-\mathrm{mm}$ filter.

827 Vero cells were transduced with lentiviral suspensions directly added to the cells.

828 After 72 hours post transduction, the medium was shifted to DMEM $5 \%$ FBS

829 containing puromycin $(20 \mu \mathrm{g} / \mathrm{ml})$ to select transduced cells.

830

831 Indirect immunofluorescence and antibodies, conventional and confocal

832 microscopy.

833 Cells were seeded onto $13 \mathrm{~mm}$ glass coverslips in 24 well plates prior to ASFV

834 infection. Then, cells were washed with PBS and fixed with $4 \%$ paraformaldehyde

835 (PFA) for 15 min. After a PBS wash, cells were then incubated with $50 \mathrm{mM} \mathrm{NH}_{4} \mathrm{Cl}$ 
836 in PBS for $10 \mathrm{~min}$. Then, coverslips were incubated in blocking buffer $(0.1 \%$

837 saponin, $0.5 \%$ BSA in PBS) for $1 \mathrm{~h}$. Coverslips were then incubated for $1 \mathrm{~h}$ in

838 specific primary antibodies diluted in blocking buffer at $37^{\circ} \mathrm{C}$. The following rabbit

839 antibodies were used: Rab7 (Cell signaling) and NPC1 (Abcam).

840 Mouse monoclonal antibodies were: p72 (1BC11, Ingenasa) and CD63 (Novus

841 biologicals). The appropriate secondary antibody conjugated to Alexa Fluor -488

842 or -594 (ThermoFisher) was used and cell nuclei was detected with TOPRO3

843 (ThermoFisher). Coverslips were mounted on glass slides using ProLong Gold

844 (ThermoFisher).

845 Cells were visualized using TCS SPE confocal microscope (Leica) with a 63X Oil

846 immersion objective. The Image acquisition was performed with a Leica

847 Application Suite Advanced Fluorescence Software (LAS AF). All the images

848 were taken with a 1024 X 1024 pixels' resolution.

849 To detect free intracellular cholesterol, coverslips were incubated with $100 \mu \mathrm{g} / \mathrm{ml}$

850 filipin (Sigma) for 1h. Filipin signal was recorded using a 390- to 415-nm-

851 wavelength excitation filter and a 450- to 470-nm-wavelength emission filter in a

852 conventional fluorescence microscopy Leica (DM RB). Image analyses were

853 performed with Leica Application Suite advanced fluorescence software (LAS AF)

854 and ImageJ software.

856 Sequence comparison

857 Sequences of $\mathrm{pE} 248 \mathrm{R}$ and $\mathrm{pE} 199 \mathrm{~L}$ proteins were selected from the NCBI data.

$858 \mathrm{NCBI}$ reference sequences for proteins of the different ASFV isolates were:

859 txid10498 for Ba71V, for the Georgia strain txid874269 was used, txid561443 for

860 the South African, txid561444 for Namibia and txid561445 for the Kenyan isolates 
861 respectively. For Vaccinia Virus (VACV) Western Reserve (WR) proteins A16L,

862 A26L, G9, and L1R, the NCBI reference: txid10254 was used.

863 BLAST was performed against the E199L protein of ASFV Ba71V, finding 25\%

864 identity with A16L fusion protein of Entomopoxvirus Mythimna separata, with

865 NCBI reference: txid1293572. This protein has a $57.3 \%$ identity with VACV WR

866 fusion protein A16L.

867 Alignments between E199L of ASFV Ba71V and A16L of VACV WR, were made 868 using the Geneious 6.0.6 program, with a standard BLOSUM62 matrix as these

869 proteins belong to a different viral family. Sequence similarities were found in the

870 alignments, which could originate a structural similarity and possibly a functional

871 homology to be explored. Alignment with the same matrix was performed

872 between the protein L1R of the VACV fusion complex and the ASFV fusion 873 protein E248R.

874 Also, alignments of the E248R protein sequences between the different ASFV

875 isolates of were made using the Geneious 6.0.6 program. BLOSUM 90 alignment

876 matrix was used, as this is the most accurate comparison matrix between

877 different isolates of the same viral species. The same was done for the E199L 878 proteins of the different ASFV isolates.

\section{Statistical analysis}

881 The experimental data were analysed with Unpaired Student $t$-test by Graph Pad

882 Prism 5 software. Values were expressed in graph bars as mean \pm sem of at least 883 three independent experiments. Statistical significance was assigned at ${ }^{*} p<0.05$, ${ }^{* *} p<0.01,{ }^{* * *} p<0.001$ and ${ }^{* * * *} p<0.0001 ;$ n.s, not significant. 


\section{Acknowledgements}

888 This research was partially supported through "La Caixa" Banking Foundation 889 award number LCF/PR/HR19/52160012, Spain; Spanish Ministry of Science and

890 Innovation, Spain RTI2018-097305-R- I00) Instituto de Salud Carlos III, ISCIII,

891 Spain FIS PI 1801007 and the European Commission, Horizon 2020 Framework 892 Programme European Union ASFVInt- ERANET-2021-0017 and VIRUSCAN 893 FETPROACT-2016- 731868.

\section{Supporting information}

896 S1 Fig. E248R and E199L structure resembles to VACV fusion machinery.

897 Comparison of the amino acid protein sequences of E248R (ASFV, Ba71V 898 isolate) with L1R (VACV WR); and E199L (ASFV Ba71V isolate) with their 899 potential equivalents in VACV A16, G9 and A26. The identical amino acids are 900 indicated in green or purple respectively, in grey the amino acids conserved 901 between 60 and $80 \%$, and the amino acids conserved less than $60 \%$ in white.

902 S2 Fig: E248R and mutants, E199L binding to NPC1. Membranes used to compose figure 3B. Dashed boxes were taken to create the western blot

904 composition showed in the figure. Membranes were revealed with mouse anti905 Flag antibody, mouse anti-HA antibody and mouse anti-tubulin.

906 S3 Fig. VACV L1R binding to NPC1. (A) Membranes used to compose figure

$9073 \mathrm{C}$ and (B) 3D. Dashed boxes were taken to create the western blot composition 908 showed in the figure. Membranes were revealed with mouse anti-Flag antibody, 909 mouse anti-HA antibody and mouse anti-tubulin. 
910 S4 Fig. Reverse Pull down E248R. Membranes used to compose figure 3F.

911 Dashed boxes were taken to create the western blot composition showed in the

912 figure. Membranes were revealed with mouse anti-Flag antibody, mouse anti-HA

913 antibody and mouse anti-tubulin.

914 S5 Fig. Reverse Pull down E199L. Membranes used to compose figure $3 G$.

915 Dashed boxes were taken to create the western blot composition showed in the

916 figure. Membranes were revealed with mouse anti-Flag antibody, mouse anti-HA

917 antibody and rat anti HSP90.

918 S6 Fig. NPC1 KO validation in Vero cells. (A) NPC1 detection in Vero or Vero

919 NPC1 KO cell lines (B) Indirect immunofluorescence depicts NPC1 in green

920 detected with a specific antibody against NPC1. (C-D) NPC1 KO cells depicts

921 dilated endosomes detected in red with the acidic probe Lysotracker. Cholesterol

922 stained with Filipin III (in blue) accumulates in dilated vesicles, similarly as occurs

923 in cells pre-treated with U18666A drug. (E) Infection of Vero and NPC1-KO-Vero

924 cells with recombinant VSV (rVSVs) pseudotyped with Ebolavirus Glycoprotein

925 (EBOV-GP) Mayinga strain or VSV-G 24 h. Cells were lysed 24 hs post-infection

926 and assayed for luciferase expression. Percentages of infectivity were

927 determined by setting the number of RLU in Vero cells to $100 \%$ for each

928 envelope.

929

930 S7 Fig. NPC1 KD impact ASFV infectivity. (A) NPC1 detection in Vero WT Scr 931 (scrambled) cells or Vero NPC1 KD (shNPC1-52) cells with a rabbit anti-NPC1

932 antibody. (B) B54GFP infection percentages at 16h in Vero WT Scr and Vero 933 NPC1 KD cells (shNPC1-52) as detected by flow cytometry. (C) ASFV genome 
copy number in Scr and Vero NPC1 KD (shNPC1-52), infected with ASFV and analysed by real-time PCR.

936

\section{References}

1. Andrés G, Charro D, Matamoros T, Dillard RS, Abrescia NGJJoBC. The cryoEM structure of African swine fever virus unravels a unique architecture comprising two icosahedral protein capsids and two lipoprotein membranes. 2020;295(1):1-12.

944 2. Liu S, Luo Y, Wang Y, Li S, Zhao Z, Bi Y, et al. Cryo-EM structure of the African swine fever virus. 2019;26(6):836-43. e3.

946 3. Wang N, Zhao D, Wang J, Zhang Y, Wang M, Gao Y, et al. Architecture of 947 African swine fever virus and implications for viral assembly. 2019;366(6465):640-4.

948 4. Rowlands RJ, Michaud V, Heath L, Hutchings G, Oura C, Vosloo W, et al. 949 African swine fever virus isolate, Georgia, 2007. 2008;14(12):1870.

950 5. Brookes V, Barrett T, Ward M, Roby J, Hernandez-Jover M, Cross E, et al. A 951 scoping review of African swine fever virus spread between domestic and free-living 952 pigs. 2020.

953 6. www.fli.de. Wild boar with African swine fever in the district of Märkisch954 Oderland 2020 [

9557 7bedin SN, Baruah A, Bora A, Dutta D, Dutta AJPI. African swine fever (ASF) 956 outbreak in India: A review of literature about the virus and its control measures. 957 2020;9(7):298-304.

958 8. Dixon LK, Stahl K, Jori F, Vial L, Pfeiffer DUJAroab. African swine fever 959 epidemiology and control. 2020;8:221-46.

960 9. Galindo I, Cuesta-Geijo MA, Hlavova K, Muñoz-Moreno R, Barrado-Gil L, 961 Dominguez J, et al. African swine fever virus infects macrophages, the natural host cells, 962 via clathrin-and cholesterol-dependent endocytosis. 2015;200:45-55.

963 10. Sánchez EG, Quintas A, Pérez-Núñez D, Nogal M, Barroso S, Carrascosa ÁL, et 964 al. African swine fever virus uses macropinocytosis to enter host cells. 965 2012;8(6):e1002754.

966 11. Cuesta-Geijo MA, Galindo I, Hernáez B, Quetglas JI, Dalmau-Mena I, Alonso 967 CJPo. Endosomal maturation, Rab7 GTPase and phosphoinositides in African swine 968 fever virus entry. 2012;7(11):e48853.

969 12. Cuesta-Geijo MÁ, Chiappi M, Galindo I, Barrado-Gil L, Muñoz-Moreno R, 970 Carrascosa JL, et al. Cholesterol flux is required for endosomal progression of African 971 swine fever virions during the initial establishment of infection. 2016;90(3):1534-43.

972 13. Qian H, Wu X, Du X, Yao X, Zhao X, Lee J, et al. Structural basis of low-pH973 dependent lysosomal cholesterol egress by NPC1 and NPC2. 2020;182(1):98-111. e18.

974 14. Carstea ED, Morris JA, Coleman KG, Loftus SK, Zhang D, Cummings C, et al. 975 Niemann-Pick C1 disease gene: homology to mediators of cholesterol homeostasis. 976 1997;277(5323):228-31.

977 15. Ikonen EJCoicb. Mechanisms of cellular cholesterol compartmentalization: recent 978 insights. 2018;53:77-83. 
979 16. Storch J, Xu ZJBeBA-M, Lipids CBo. Niemann-Pick C2 (NPC2) and intracellular 980 cholesterol trafficking. 2009;1791(7):671-8.

981 17. Gong X, Qian H, Zhou X, Wu J, Wan T, Cao P, et al. Structural insights into the 982 Niemann-Pick C1 (NPC1)-mediated cholesterol transfer and Ebola infection. 983 2016;165(6):1467-78.

984 18. Wang H, Shi Y, Song J, Qi J, Lu G, Yan J, et al. Ebola viral glycoprotein bound 985

986

987

988

989 to its endosomal receptor Niemann-Pick C1. 2016;164(1-2):258-68.

19. Lu F, Liang Q, Abi-Mosleh L, Das A, De Brabander JK, Goldstein JL, et al. Identification of NPC1 as the target of U18666A, an inhibitor of lysosomal cholesterol export and Ebola infection. 2015;4:e12177.

990 Ebola virus glycoprotein complexes with tricyclic antidepressant and antipsychotic drugs. 991 2018;61(11):4938-45.

992 21. Ballout RA, Sviridov D, Bukrinsky MI, Remaley ATJTFJ. The lysosome: A 993 potential juncture between SARS-CoV-2 infectivity and Niemann-Pick disease type C, 994 with therapeutic implications. 2020;34(6):7253-64.

995 22. Garcia-Dorival I, Cuesta-Geijo MA, Barrado-Gil L, Galindo I, Urquiza J, Del 996 Puerto A, et al. Identification of NPC1 as a novel SARS-CoV-2 intracellular target. 2020. 23. Khan I, Katikaneni DS, Han Q, Sanchez-Felipe L, Hanada K, Ambrose RL, et al. Modulation of hepatitis $\mathrm{C}$ virus genome replication by glycosphingolipids and fourphosphate adaptor protein 2. 2014;88(21):12276-95.

1000 24. Liang X-D, Zhang Y-N, Liu C-C, Chen J, Chen X-N, Baloch AS, et al. U18666A 1001 inhibits classical swine fever virus replication through interference with intracellular 1002 cholesterol trafficking. 2019;238:108436.

1003 25. Lucifora J, Esser K, Protzer UJAr. Ezetimibe blocks hepatitis B virus infection after virus uptake into hepatocytes. 2013;97(2):195-7.

26. Osuna-Ramos JF, Reyes-Ruiz JM, del Ángel RMJFic, microbiology i. The role of host cholesterol during flavivirus infection. 2018;8:388.

27. Sainz B, Barretto N, Martin DN, Hiraga N, Imamura M, Hussain S, et al. Identification of the Niemann-Pick $\mathrm{C} 1$-like 1 cholesterol absorption receptor as a new hepatitis C virus entry factor. 2012;18(2):281-5.

1010 28. Stoeck IK, Lee J-Y, Tabata K, Romero-Brey I, Paul D, Schult P, et al. Hepatitis 1011 C virus replication depends on endosomal cholesterol homeostasis. 2018;92(1).

1012 29. Sturley SL, Rajakumar T, Hammond N, Higaki K, Márka Z, Márka S, et al. 1013 Potential COVID-19 therapeutics from a rare disease: weaponizing lipid dysregulation to 1014 combat viral infectivity. 2020;61(7):972-82.

1015 30. Tang Y, Leao IC, Coleman EM, Broughton RS, Hildreth JEJJov. Deficiency of 1016 niemann-pick type C-1 protein impairs release of human immunodeficiency virus type 1 1017 and results in Gag accumulation in late endosomal/lysosomal compartments. 1018 2009;83(16):7982-95.

1019 31. Wang H, Perry JW, Lauring AS, Neddermann P, De Francesco R, Tai AWJG. 1020 Oxysterol-binding protein is a phosphatidylinositol 4-kinase effector required for HCV 1021 replication membrane integrity and cholesterol trafficking. 2014;146(5):1373-85. e11.

1022 32. Wichit S, Hamel R, Bernard E, Talignani L, Diop F, Ferraris P, et al. Imipramine 1023 inhibits chikungunya virus replication in human skin fibroblasts through interference with intracellular cholesterol trafficking. 2017;7(1):1-12.

33. Hernáez B, Guerra M, Salas ML, Andrés GJPp. African swine fever virus undergoes outer envelope disruption, capsid disassembly and inner envelope fusion before core release from multivesicular endosomes. 2016;12(4):e1005595. 
1028 34. Matamoros T, Alejo A, Rodríguez JM, Hernáez B, Guerra M, Fraile-Ramos A, et 1029 al. African Swine Fever Virus Protein pE199L Mediates Virus Entry by Enabling 1030 Membrane Fusion and Core Penetration. 2020;11(4):e00789-20.

1031 35. Rodríguez I, Nogal ML, Redrejo-Rodríguez M, Bustos MJ, Salas MLJJov. The 1032 African swine fever virus virion membrane protein $\mathrm{pE} 248 \mathrm{R}$ is required for virus infectivity and an early postentry event. 2009;83(23):12290-300.

36. Maurer-Stroh S, Eisenhaber FJTim. Myristoylation of viral and bacterial proteins. 2004;12(4):178-85.

protein disulfide bond formation encoded by poxviruses. 2002;99(10):6667-72.

1038 38. Rodríguez I, Redrejo-Rodríguez M, Rodríguez JM, Alejo A, Salas J, Salas

1039 MLJJov. African swine fever virus $\mathrm{pB} 119 \mathrm{~L}$ protein is a flavin adenine dinucleotide1040 linked sulfhydryl oxidase. 2006;80(7):3157-66.

1041 39. Sun H, Jenson J, Dixon LK, Parkhouse RMEJJogv. Characterization of the 1042 African swine fever virion protein j18L. 1996;77(5):941-6.

1043 40. Carette JE, Raaben M, Wong AC, Herbert AS, Obernosterer G, Mulherkar N, et 1044 al. Ebola virus entry requires the cholesterol transporter Niemann-Pick C1. 1045 2011;477(7364):340-3.

1046 41. Miller EH, Obernosterer G, Raaben M, Herbert AS, Deffieu MS, Krishnan A, et 1047 al. Ebola virus entry requires the host-programmed recognition of an intracellular 1048 receptor. 2012;31(8):1947-60.

1049 42. Cuesta-Geijo MÁ, Barrado-Gil L, Galindo I, Muñoz-Moreno R, Alonso CJV. 1050 Redistribution of endosomal membranes to the African swine fever virus replication site. $10512017 ; 9(6): 133$.

1052 43. Kwon HJ, Abi-Mosleh L, Wang ML, Deisenhofer J, Goldstein JL, Brown MS, et 1053 al. Structure of N-terminal domain of NPC1 reveals distinct subdomains for binding and 1054 transfer of cholesterol. 2009;137(7):1213-24.

1055 44. Naureckiene S, Sleat DE, Lackland H, Fensom A, Vanier MT, Wattiaux R, et al. 1056 Identification of $\mathrm{HE} 1$ as the second gene of Niemann-Pick $\mathrm{C}$ disease. 1057 2000;290(5500):2298-301.

1058 45. Sleat DE, Wiseman JA, El-Banna M, Price SM, Verot L, Shen MM, et al. Genetic 1059 evidence for nonredundant functional cooperativity between NPC1 and NPC2 in lipid 1060 transport. 2004;101(16):5886-91.

1061 46. Iyer LM, Aravind L, Koonin EVJJov. Common origin of four diverse families of 1062 large eukaryotic DNA viruses. 2001;75(23):11720-34.

1063 47. Moss B, editor Membrane fusion during poxvirus entry. Seminars in cell \& 1064 developmental biology; 2016: Elsevier.

1065 48. Song J, Li K, Li T, Zhao G, Zhou S, Li H, et al. Screening of PRRSV-and ASFV1066 encoded proteins involved in the inflammatory response using a porcine iGLuc reporter. 1067 2020;285:113958.

1068 49. Bao Y-J, Qiu J, Rodríguez F, Qiu H-JJb. The genetic variation landscape of 1069 African swine fever virus reveals frequent positive selection on amino acid replacements. 10702020.

1071 50. Lee JE, Saphire EOJFv. Ebolavirus glycoprotein structure and mechanism of 1072 entry. 2009;4(6):621-35.

1073 51. Côté M, Misasi J, Ren T, Bruchez A, Lee K, Filone CM, et al. Small molecule 1074 inhibitors reveal Niemann-Pick C1 is essential for Ebola virus infection. 1075 2011;477(7364):344-8. 
1076 52. Ko DC, Gordon MD, Jin JY, Scott MPJMbotc. Dynamic movements of organelles 1077 containing Niemann-Pick C1 protein: NPC1 involvement in late endocytic events. $1078 \quad 2001 ; 12(3): 601-14$.

1079 53. Watari H, Blanchette-Mackie EJ, Dwyer NK, Glick JM, Patel S, Neufeld EB, et 1080 al. Niemann-Pick C1 protein: obligatory roles for N-terminal domains and lysosomal targeting in cholesterol mobilization. 1999;96(3):805-10.

54. Altmann SW, Davis HR, Zhu L-j, Yao X, Hoos LM, Tetzloff G, et al. NiemannPick C1 Like 1 protein is critical for intestinal cholesterol absorption. 2004;303(5661):1201-4.

1087 56. Poh MK, Shui G, Xie X, Shi P-Y, Wenk MR, Gu FJAr. U18666A, an intra-cellular 1088 cholesterol transport inhibitor, inhibits dengue virus entry and replication. 1089 2012;93(1):191-8.

1090 57. Shoemaker CJ, Schornberg KL, Delos SE, Scully C, Pajouhesh H, Olinger GG, et 1091 al. Multiple cationic amphiphiles induce a Niemann-Pick C phenotype and inhibit Ebola 1092 virus entry and infection. 2013;8(2):e56265.

1093 58. Lasala F, García-Rubia A, Requena C, Galindo I, Cuesta-Geijo MA, GarcíaDorival I, et al. Identification of potential inhibitors of protein-protein interaction useful to fight against Ebola and other highly pathogenic viruses. 2021;186:105011. 59. Enjuanes L, Carrascosa A, Moreno M, Vinuela EJJoGV. Titration of African swine fever (ASF) virus. 1976;32(3):471-7. 60. Hernaez B, Escribano JM, Alonso CJV. Visualization of the African swine fever virus infection in living cells by incorporation into the virus particle of green fluorescent protein-p54 membrane protein chimera. 2006;350(1):1-14.

1101 61. Barrado-Gil L, Galindo I, Martínez-Alonso D, Viedma S, Alonso CJPO. The 1102 ubiquitin-proteasome system is required for African swine fever replication. 1103 2017;12(12):e0189741.

1104 62. Kobayashi T, Beuchat M-H, Lindsay M, Frias S, Palmiter RD, Sakuraba H, et al. 1105 Late endosomal membranes rich in lysobisphosphatidic acid regulate cholesterol transport. 1999;1(2):113-8.

1107 63. Liscum L, Faust JRJJoBC. The intracellular transport of low density lipoprotein1108 derived cholesterol is inhibited in Chinese hamster ovary cells cultured with 3- $\beta$-[21109 (diethylamino) ethoxy] androst-5-en-17-one. 1989;264(20):11796-806.

1110 64. https://www.deskgen.com/guidebook/advanced.html.

1111 65. King DP, Reid SM, Hutchings GH, Grierson SS, Wilkinson PJ, Dixon LK, et al. 1112 Development of a TaqMan ${ }^{\circledR}$ PCR assay with internal amplification control for the detection of African swine fever virus. 2003;107(1):53-61. 